\title{
Article
}

\section{Suppressing the Shuttle Effect and Dendrite Growth in Lithium-Sulfur Batteries}

Jianan Wang, Shanshan Yi, Jianwei Liu, Sun Shiyi, Yunpeng Liu, Duowen Yang, Kai Xi, Guoxin Gao, Amr Abdelkader, Wei Yan, Shujiang Ding, and Ramachandran Vasant Kumar

ACS Nano, Just Accepted Manuscript • DOI: 10.1021/acsnano.0c02241 • Publication Date (Web): 07 Jul 2020

Downloaded from pubs.acs.org on July 7, 2020

\section{Just Accepted}

"Just Accepted" manuscripts have been peer-reviewed and accepted for publication. They are posted online prior to technical editing, formatting for publication and author proofing. The American Chemical Society provides "Just Accepted" as a service to the research community to expedite the dissemination of scientific material as soon as possible after acceptance. "Just Accepted" manuscripts appear in full in PDF format accompanied by an HTML abstract. "Just Accepted" manuscripts have been fully peer reviewed, but should not be considered the official version of record. They are citable by the Digital Object Identifier (DOI®). "Just Accepted" is an optional service offered to authors. Therefore, the "Just Accepted" Web site may not include all articles that will be published in the journal. After a manuscript is technically edited and formatted, it will be removed from the "Just Accepted" Web site and published as an ASAP article. Note that technical editing may introduce minor changes to the manuscript text and/or graphics which could affect content, and all legal disclaimers and ethical guidelines that apply to the journal pertain. ACS cannot be held responsible for errors or consequences arising from the use of information contained in these "Just Accepted" manuscripts. 
Suppressing the Shuttle Effect and Dendrite Growth in Lithium-Sulfur

\section{Batteries}

Jianan Wang, ${ }^{\dagger} \S$ Shanshan Yi,,$^{\dagger}$ Jianwei Liu, ${ }^{\dagger}$ Shiyi Sun, ${ }^{\dagger}$ Yunpeng Liu, ${ }^{\dagger}$ Duowen Yang, ${ }^{\dagger}$ Kai

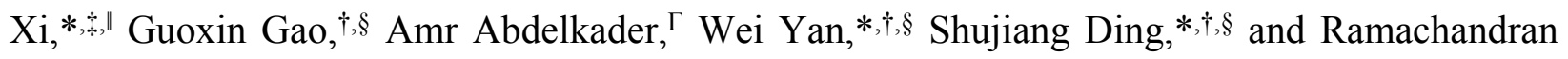
Vasant Kumar"

†Department of Environmental Science and Engineering, Department of Applied Chemistry, School of Chemistry, MOE Key Laboratory for Nonequilibrium Synthesis and Modulation of Condensed Matter, State Key Laboratory for Electrical Insulation and Power Equipment, Xi'an Jiaotong University, Xi’an 710049, China.

\#Department of Engineering, University of Cambridge, Cambridge, CB3 0FA, United Kingdom.

"Department of Materials Science and Metallurgy, University of Cambridge, Cambridge, CB3 OFS, United Kingdom.

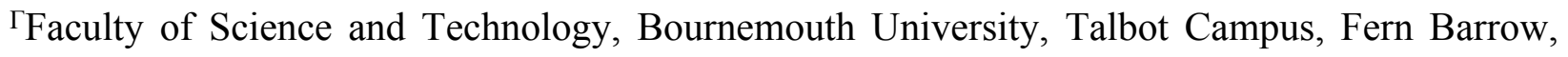
Poole, BH12 5BB, United Kingdom.

$\S$ Xi'an Jiaotong University \& Shaanxi Quantong Joint Research Institute of New Energy Vehicles Power

*E-mail: dingsj@xjtu.edu.cn (S. Ding); yanwei@xjtu.edu.cn (W. Yan); kx210@cam.ac.uk (K. $\mathrm{Xi)}$ 


\begin{abstract}
Practical applications of lithium-sulfur batteries are simultaneously hindered by two serious problems occurring separately in both electrodes, namely, the shuttle effects of lithium polysulfides and the uncontrollable growth of lithium dendrites. Herein, to explore a facile integrated approach to tackle both problems as well as guarantee the efficient charge transfer, we used two-dimension hexagonal $\mathrm{VS}_{2}$ flakes as the building blocks to assemble nanotowers on the separators, forming symmetrical double-side-modified polypropylene separator without blocking the membrane pores. Benefiting from the "sulfiphilic" and "lithiophilic" properties, high interfacial electronic conductivity and unique hexagonal towerform nanostructure, the D-HVS@PP separator not only guarantee the effective suppression of lithium polysulfide shuttle and the rapid ion/electron transfer, but also realize the uniform and stable lithium nucleation and growth during cycling. Hence, just at the expense of an $11 \%$ increase in the separator weight $\left(0.14 \mathrm{mg} \mathrm{cm}^{-2}\right)$, D-HVS@PP separator delivers an over 16 times higher initial areal capacity $\left(8.3 \mathrm{mAh} \mathrm{cm}^{-2}\right)$ than conventional PP separator $\left(0.5 \mathrm{mAh} \mathrm{cm}^{-2}\right)$ under high sulfur-loading condition $\left(9.24 \mathrm{mg} \mathrm{cm}^{-2}\right)$. Even when used under a low electrolyte/sulfur ratio of $4 \mathrm{~mL} \mathrm{~g}^{-1}$ and a practically relevant N/P ratio of 1.7, D-HVS@PP separator still enabled stable cycling with a high cell-level gravimetric energy density. The potentials in broader applications (Li-S pouch battery and $\mathrm{Li}-\mathrm{LiFePO}_{4}$ battery) and the promising commercial prospect (large-scale production and recyclability) of the developed separator are also demonstrated.
\end{abstract}

KEYWORDS: lithium-sulfur batteries, amphiphilic, separator, lithium dendrites, recyclable, shuttle effect 
The rapid development of portable electronic devices, electric vehicles and smart grids has evoked the ever-increasing demand for high-energy-density energy storage systems with sustainable electrochemical performances. ${ }^{1-3}$ Lithium-sulfur ( $\left.\mathrm{Li}-\mathrm{S}\right)$ batteries are regarded as a potential alternative to current state-of-the-art Li-ion batteries owing to their high theoretical capacity (1675 $\mathrm{mAh}^{-1}$ of sulfur) and energy density (2600 $\left.\mathrm{Wh} \mathrm{kg}^{-1}\right)$, low cost and environmental friendliness. ${ }^{4-6}$ Despite such a bright perspective, the practical implementation of Li-S batteries is still facing some tough challenges. In terms of the sulfur cathode, the severe "shuttling effect" of the dissolved intermediate lithium polysulfides $\left(\mathrm{Li}_{2} \mathrm{~S}_{\mathrm{x}}\right)$ gives rise to low active sulfur utilization, low coulombic efficiency and rapid capacity decay; $;^{7-9}$ For the lithium anode, the uncontrollable growth of lithium "dendrites" on the surface of lithium metal induces a series of adverse effects, such as the evolution of "dead" lithium, unstable solid electrolyte interphase (SEI), increased polarization and even explosion hazards. ${ }^{10,11}$ Due to the disparate reaction mechanism and different physicochemical characteristics of the sulfur cathode and the lithium anode, synchronously suppressing the shuttle effect and the dendrite growth during long-term cycling has become a formidable technical challenge for the practical application of Li-S batteries.

To date, several approaches have been developed to address these issues, including designing cathode host materials, ${ }^{12,}{ }^{13}$ inserting interlayer, ${ }^{14}$ substituting binders ${ }^{15}$ and modifying the separator or the lithium anode. ${ }^{16-19}$ The majority of these technical strategies only focus on one part of the cell. Adopting an integrated approach that can simultaneously solve the lithium dendrites and $\mathrm{Li}_{2} \mathrm{~S}_{\mathrm{x}}$ shuttle effect maybe the way forward to make Li-S batteries achieve the commercial realization. Separators, as the 
essential medium directly contacting and interacting with both the anode and the cathode, play a vital role in the battery system. ${ }^{20-23}$ Functionalizing both sides of commercially available separators is considered to be a facile/effective strategy in controlling the interfacial reactions of both the multielectron conversion of sulfur/polysulfide and the lithium deposition/dissolution, further boosting the overall battery performance. ${ }^{24-26}$ For this purpose, some asymmetric separator structures have been developed recently to satisfy the distinct requirements of both the cathode and the anode sides. ${ }^{20,27,28}$ However, the majority of these separators has shown difficulties to maintain the inherent pore structures of the separator itself during the charging/discharging process, representing a constraint for the high-flux $\mathrm{Li}^{+}$diffusion. ${ }^{20,29}$ In addition, the complicated design of the asymmetrical separator inevitably increases the difficulty for the separator commercialization. Based on the above analysis, it would be appealing to rationally select and design a multifunctional material using for separator modification, which could simultaneously meet distinct demands of the anode and cathode in Li-S batteries as well as guarantee the smooth ion diffusion.

Vanadium disulfide $\left(\mathrm{VS}_{2}\right)$ is one of the transition-metal dichalcogenides (TMDs) that has attracted increasing interests in the fields of electrochemical energy storage in recent years because of its unique chemical/physical characteristics, intrinsic metallic behavior and two-dimensional (2D) layered structure. ${ }^{30-32}$ Cui et al. ${ }^{30}$ have reported that commercial $\mathrm{VS}_{2}$ could exhibit higher capacity and better cycling stability compared with other TMDs materials ( $\mathrm{TiS}_{2}, \mathrm{CoS}_{2}, \mathrm{Ni}_{3} \mathrm{~S}_{2}, \mathrm{SnS}_{2}$ and $\mathrm{FeS}$ ) and graphite when used as the cathode host material in Li-S batteries. They attributed the performance enhancement to the high conductivity, strong interaction with $\mathrm{Li}_{2} \mathrm{~S}_{\mathrm{x}}$, easy $\mathrm{Li}$-ion transport and excellent 
catalyzing reduction/oxidation capability of $\mathrm{VS}_{2}$. All these superiorities signify that $\mathrm{VS}_{2}$ deserves specific attention as a promising functional material not only for the cathode but also as a modifier for the separator, an area which hardly been investigated in the literature. Furthermore, the performance of $\mathrm{VS}_{2}$ at the anode side, particularly the interaction at the anode/separator interfaces remain unexplored, despite the clear physical advantageous. Also, coating $\mathrm{VS}_{2}$ on the separators is a more simple and low-cost binderfree modification strategy than coating the lithium metal surface. The facile coating process on the polymer separator would facilitate the practical application and commercial production of Li-S batteries. ${ }^{33,34}$

Herein, a recyclable $\mathrm{VS}_{2}$ hexagonal nanotowers (HVS) double-side-modified commercial polypropylene (PP) separator (D-HVS@PP separator) was fabricated via a single-step hydrothermal method and subsequent vacuum filtration (Figure S1). The fabricated D-HVS@PP separator kept both the "sulfiphilic" and "lithiophilic" features, which can simultaneously trap $\mathrm{Li}_{2} \mathrm{~S}_{\mathrm{x}}$ and suppress lithium dendrites. In addition, due to the hexagonal tower-form nanostructure of the HVS, the D-HVS@PP separator also exhibited a high-flux lithium-ion diffusion with improved mechanical strength. As a result, the elaborate separator delivered high charge/discharge capacity and stable cycling performance in Li-S batteries, even under high sulfur loading/lean-electrolyte conditions with the controlled N/P ratio and when used in the pouch Li-S and lithium metal battery systems.

\section{RESULTS AND DISCUSSION}


C
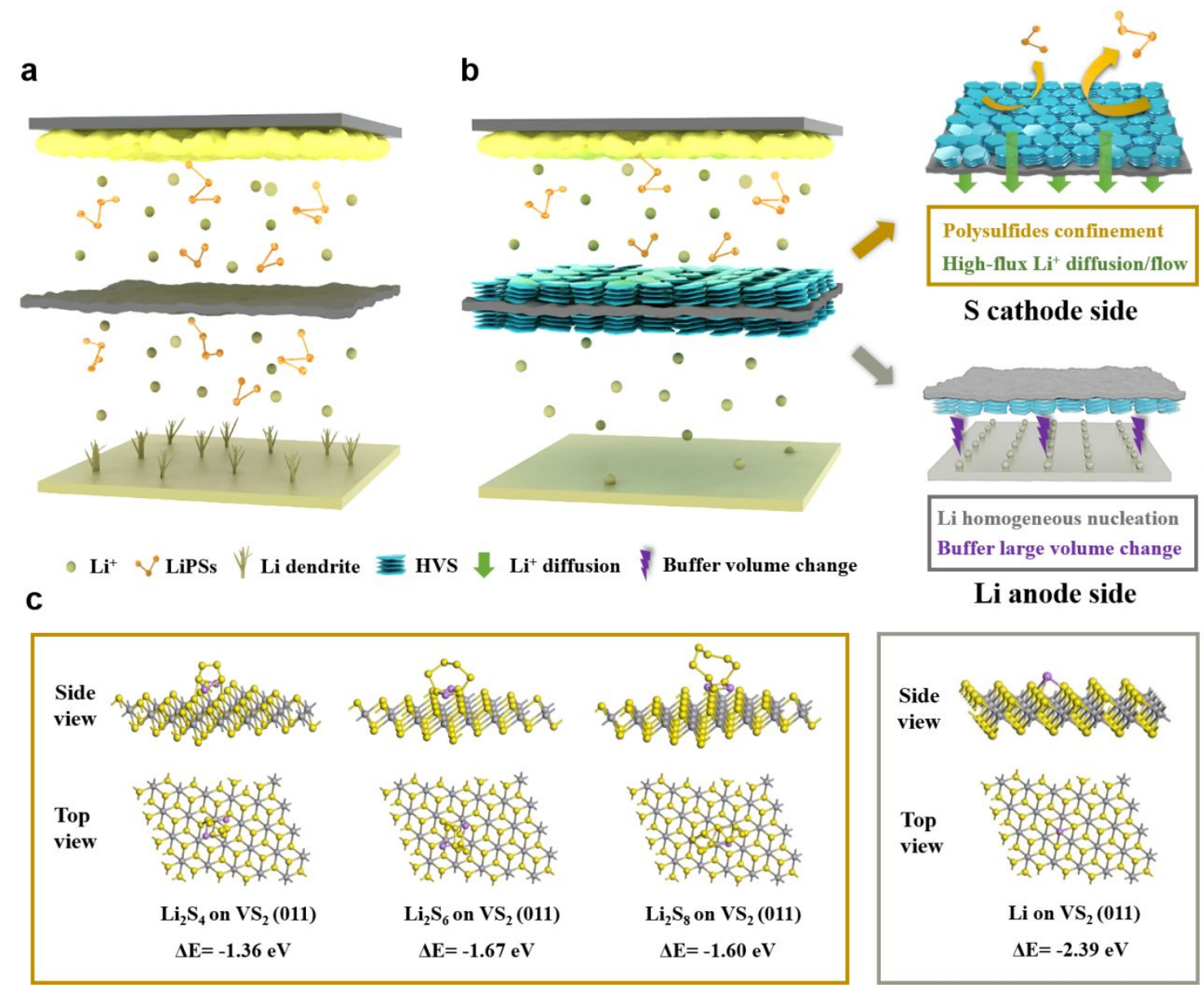

Polysulfides confinement High-flux $\mathrm{Li}^{+}$diffusion/flow

$\mathrm{S}$ cathode side

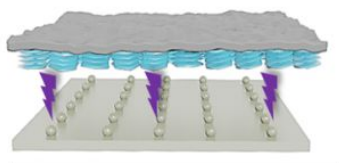

Li homogeneous nucleation Buffer large volume change

Li anode side

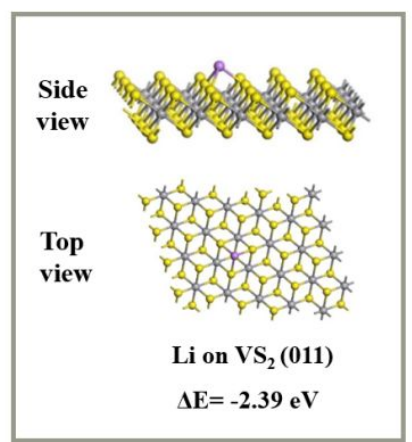

Figure 1. Working mechanism and theory simulation. Schematic illustrations of the working principle of the Li-S battery with (a) PP and (b) D-HVS@PP separator. (c) Density functional theory (DFT) calculation of the absorption energies of $\mathrm{Li}_{2} \mathrm{~S}_{4}, \mathrm{Li}_{2} \mathrm{~S}_{6}, \mathrm{Li}_{2} \mathrm{~S}_{8}$ and $\mathrm{Li}$ on $\mathrm{VS}_{2}$ (011) facet (Vienna $A b$-initio Simulation Package (VASP)).

As illustrated in Figure 1, the commonly employed PP separator in conventional Li-S batteries guarantees the primary functions for the efficient migration of lithium ions and the isolation of the counter electrodes. However, it can hardly restrain the polysulfide shuttle, and Li dendrites growth (Figure 1a). ${ }^{16,}{ }^{24}$ In the current work, an "amphiphilic" HVS material was introduced onto both sides of the PP separator, simultaneously realizing the distinct functionalities for the anode and cathode (Figure 1b). In the sulfur cathode side, the HVS can effectively prevent the shuttled $\mathrm{Li}_{2} \mathrm{~S}_{\mathrm{x}}$ from passing through the 
separator due to the strong chemical interactions. The designed layer-by-layer stacked nanostructure of the HVS also creates the abundant channels and spaces for high-flux ion diffusion/flow and provides enough exposed active sites for polysulfide adsorption. In addition, the intrinsic metallic nature of the HVS can further reduce the interfacial resistance between the electrode and the separator, enabling low polarization and fast sulfur conversion kinetics ${ }^{35}$. In the lithium anode side, the strong lithiophilic ability and high electronic conductivity of the HVS can induce ions/electrons to uniformly distribute at anode/separator interfaces, avoiding the formation of lithium dendrites caused by the local charge concentration. ${ }^{36-38}$ At the same time, the stable hexagonal tower-shaped architecture of the HVS is also beneficial to buffer the large volume change of lithium metal under deep cycling and serves as a physical shield to resist the lithium dendrites growth. ${ }^{18,39}$ Hence, the D-HVS@PP separator can boost an obvious enhancement in battery performance, and the detailed reasons have been analyzed and discussed in the following sections.

The growth process and structural characterizations of the HVS were investigated in Figure S2-6. Firstly, the scanning electron microscopy (SEM) images at various stages of the hydrothermal process are displayed in Figure S2, where the possible formation mechanism of the $\mathrm{VS}_{2}$ hexagonal nanotowers is also schematically illustrated. PVP, as a surfactant, is playing a crucial role with dual functions of the hexagonal tower-shaped nanostructure. ${ }^{40}$ First, it serves as a linking agent to bridge adjacent $\mathrm{VS}_{2}$ nanoflakes together, leading to the self-assembly of the nanoflake subunits along the c-axis. Second, it plays a vital role on controlling the size and morphology of each $\mathrm{VS}_{2}$ nanoflake along the ab-plane, leading to the formation of a perfect hexagonal nanostructure instead of the 
conventional $\mathrm{VS}_{2}$ microflowers (MVS). ${ }^{32,41,42}$ The high-resolution transmission electron microscopy (HRTEM), X-ray diffraction (XRD) and X-ray photoelectron spectroscopy (XPS) analysis in Figure S3-5, showed that both HVS and MVS samples possessed the same crystalline phase (JCPDS No. 89-1640), valence state and chemical composition, belonging to the typical $\mathrm{VS}_{2}$ structure. However, the higher intensity of the XRD peaks and the perfect interlayer structure appears in the HRTEM images suggests that HVS is of longer crystalline order. The HVS exhibited a Brunauer-Emmett-Teller (BET) surface area of $31.4 \mathrm{~m}^{2} \mathrm{~g}^{-1}$, which is over 5 times higher than the MVS $\left(5.8 \mathrm{~m}^{2} \mathrm{~g}^{-1}\right)$, since the multi-layered tower-like nanostructure was conducive to the exposure of more micro/mesopores $(10 \sim 70 \AA$ ) (Figure S6). The higher surface area allows the separator to store more electrolyte and contribute more active sites, facilitating high-flux lithium-ion diffusion and efficient interfacial reactions..$^{43,44}$

In addition, to evaluate the amphiphilic functional property of the HVS separator, the absorption energies of $\mathrm{VS}_{2}(011)$ main facet towards the soluble $\mathrm{Li}_{2} \mathrm{~S}_{\mathrm{x}}$ and metallic lithium were calculated by density functional theory (DFT) simulations. As shown in Figure 1c, the binding energies between $\mathrm{VS}_{2}(011)$ facet and $\mathrm{Li}_{2} \mathrm{~S}_{4}, \mathrm{Li}_{2} \mathrm{~S}_{6}$ and $\mathrm{Li}_{2} \mathrm{~S}_{8}$ were $1.36,-1.67$ and $-1.60 \mathrm{eV}$, respectively, much higher than that between graphene and $\mathrm{Li}_{2} \mathrm{~S}_{4}$ $(-0.56 \mathrm{eV})$ (Figure S7). This confirmed that $\mathrm{VS}_{2}$ possesses strong absorption ability for $\mathrm{Li}_{2} \mathrm{~S}_{\mathrm{x}}$, especially longer-chained $\mathrm{Li}_{2} \mathrm{~S}_{6}$ and $\mathrm{Li}_{2} \mathrm{~S}_{8}$. Long-chained $\mathrm{Li}_{2} \mathrm{~S}_{\mathrm{x}}$ are easier to dissolve and shuttle in the electrolyte and result in the rapid capacity decay of Li-S batteries. $^{7}$ Notably, $\mathrm{VS}_{2}$ also showed high chemical affinity $(-2.39 \mathrm{eV})$ for metallic lithium because of the strong interaction between Li and S atoms. This "sulfiphilic" and 
"lithiophilic" property emphasizes the potential ability of $\mathrm{VS}_{2}$ to trap $\mathrm{Li}_{2} \mathrm{~S}_{\mathrm{x}}$ and suppress lithium dendrites simultaneously.

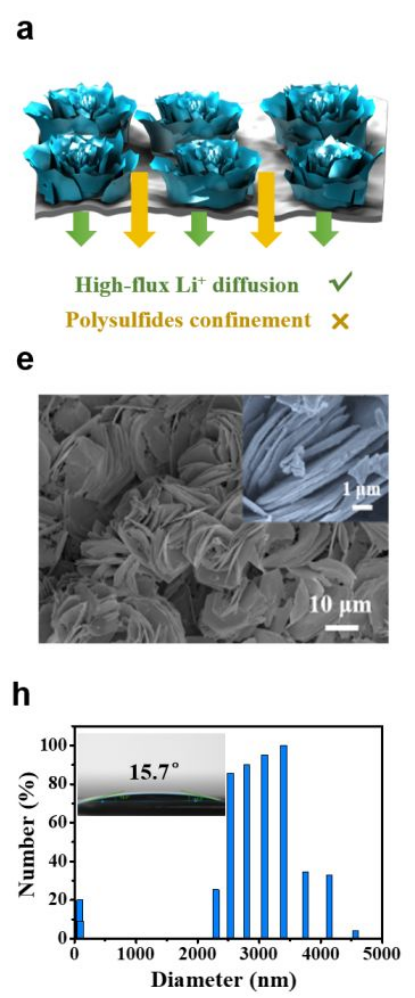

b

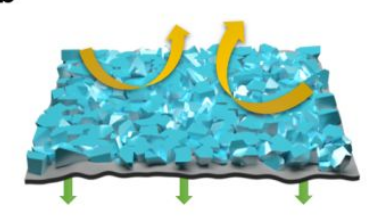

High-flux $\mathrm{Li}^{+}$diffusion $\times$ Polysulfides confinement $\checkmark$ f

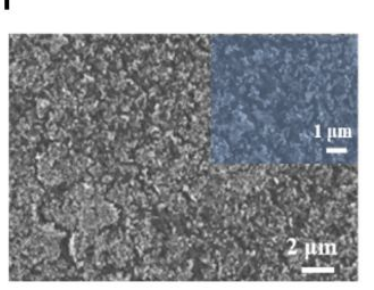

i

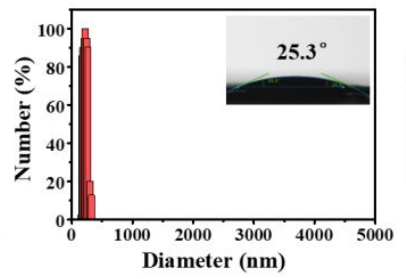

C

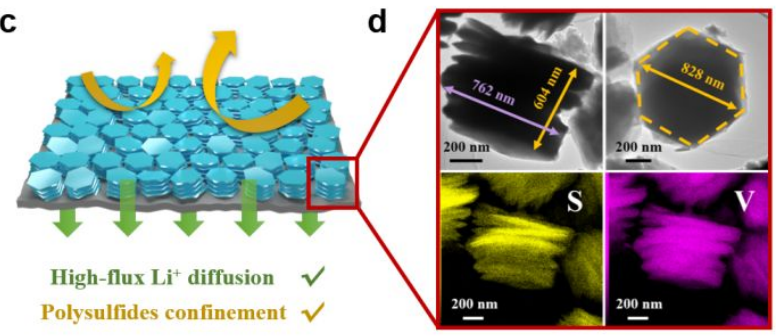

g
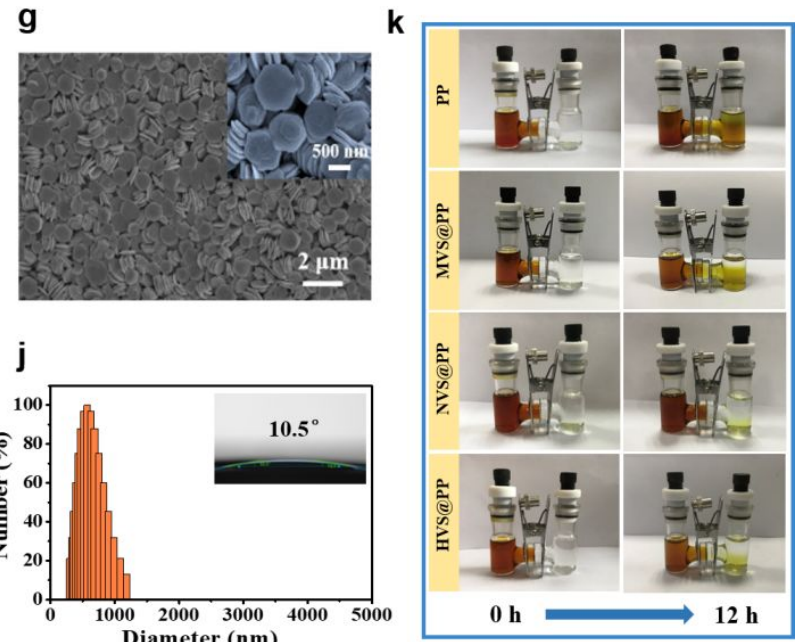

Figure 2. Functional description of different $V S_{2}$ modified separators on sulfur cathode side. Functional illustrations of the (a) MVS@PP, (b) NVS@PP and (c) HVS@PP separators on sulfur cathode side. (d) Transmission electron microscopy (TEM) images and element distribution of the $\mathrm{VS}_{2}$ hexagonal nanotowers. SEM images of (e) MVS@PP, (f) NVS@PP and (g) HVS@PP separators. Particle size distributions and contact angle images (inset) for (h) MVS@PP, (i) NVS@PP and (j) HVS@PP separators, Li-S electrolyte as a test liquid was used in contact angle tests. (k) Diffusion tests of $\mathrm{Li}_{2} \mathrm{~S}_{6}$ with PP, MVS@PP,NVS@PP and HVS@PP separators.

Before verifying the performance of the double-sided treated separator (D-HVS@PP) for the sulfur cathode or the lithium anode, a single-sided HVS@PP separator was first 
tested to investigate the reactions at one electrode and eliminate the possible influence from counter electrode. Firstly, three different $\mathrm{VS}_{2}$ modified separators (MVS@PP, VS 2 nano-bulks (NVS)@PP and HVS@PP separators) were compared. As shown in Figure 2a, e and h, the MVS@PP separator is beneficial to achieve high-flux lithium-ion diffusion, due to the high electrolyte affinity of $\mathrm{VS}_{2}$ itself and the large particle sizes (diameter 2000 5000 nm) of the MVS structure. As a result, the MVS@PP separator exhibited a much smaller contact angle $\left(15.7^{\circ}\right)$ compared with the pure PP separator $\left(37.0^{\circ}\right.$ ) (Figure S8a). However, the MVS@PP separator can hardly resist the $\mathrm{Li}_{2} \mathrm{~S}_{\mathrm{x}}$ diffusion because of the large microporous gaps among particles and low surface area of MVS (Figure 2k). Also, the flower-shaped structure and the large particle sizes reduce the contact area between MVS and the PP substrate, leading to the uneven distribution and weak adhesive strength between the components of the MVS@PP (Figure S8b). The second tested separator was NVS@PP (Figure 2b, f, i and k and Figure S9), in which NVS were obtained by directly sonicating the HVS. The NVS particles were less than $500 \mathrm{~nm}$ in diameter, which means it could be densely loaded onto the PP separator and effectively suppress $\mathrm{Li}_{2} \mathrm{~S}_{\mathrm{x}}$ shuttle. However, NVS particles formed a blocking layer on the PP substrate that significantly lowers the electrolyte wettability and hinders the lithiumion diffusion/flow. For HVS@PP separators, it can be seen that most of the nanotowers are aligned vertically on the separator with the hexagonal layers parallel to the surface (Figure 2g and Figure S10), which may be due to the more stable hexagonal 2D planes and larger plane/height ratio of the as-prepared $\mathrm{VS}_{2}$ nanotowers. ${ }^{45,46}$ Due to the moderate particle sizes (400 1200 nm), well-designed hexagonal tower-like structure and the high surface area of the HVS, HVS@PP separators (Figure 2c, d, g and j) are able to guarantee 
the adequate contact area and strong electrostatic interaction for HVS to be tightly linked to the separator (Figure S8b-d). ${ }^{45,} 47$ HVS also provided abundant tiny channels on the separator surface for both lithium-ion diffusion/flow and $\mathrm{Li}_{2} \mathrm{~S}_{\mathrm{x}}$ suppression (Figure $2 \mathrm{k}$ ).

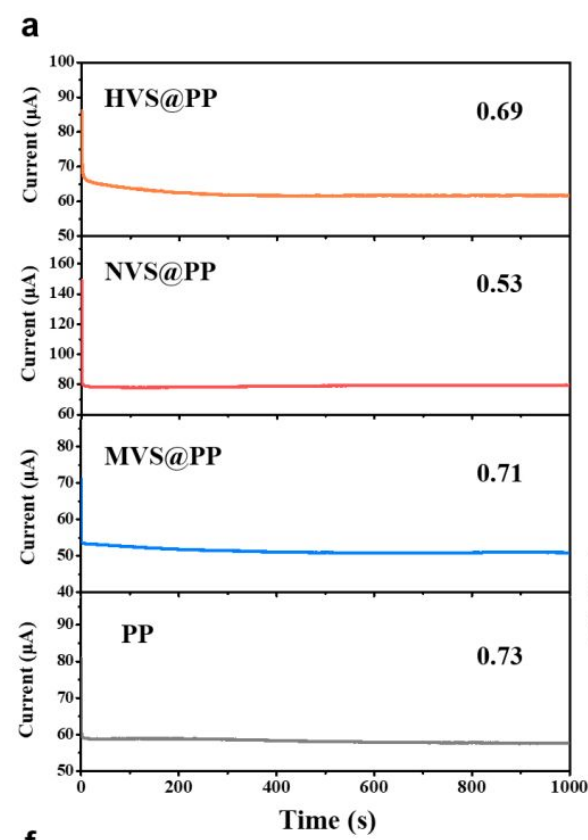

f

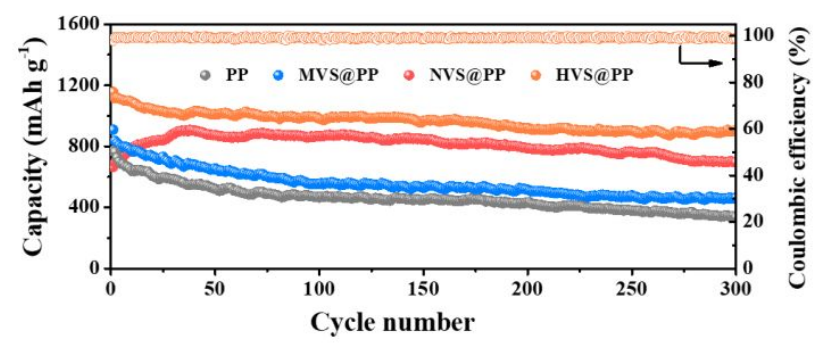

b
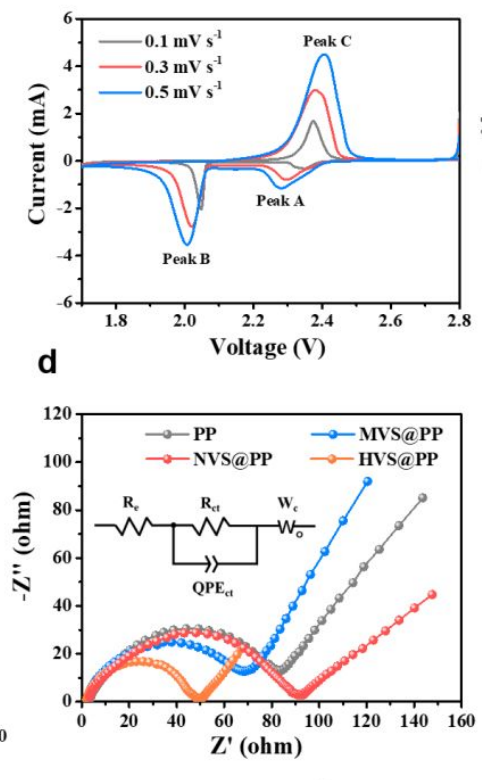

g

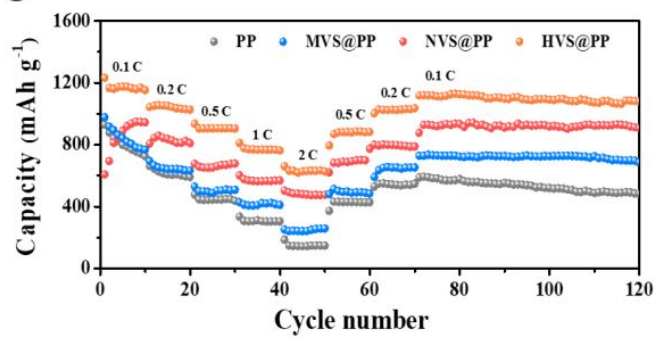

Figure 3. Electrochemical performance towards sulfur cathode side. (a) Lithium-ion transference numbers for the PP,MVS@PP, NVS@PP and HVS@PP separators tested by Li || Li symmetric cells. (b) CV plots of the HVS@PP separator at various scan rates within a potential window of $1.7 \mathrm{~V}-2.8 \mathrm{~V}\left(\mathrm{vs} . \mathrm{Li} / \mathrm{Li}^{+}\right)$. (c) Values of $\mathrm{CV}$ peak current $\left(\mathrm{I}_{\mathrm{p}}\right) /$ square root of the scan rates $\left(v^{0.5}\right)$ for the four different separators in the first (peak $A: S_{8} \rightarrow \operatorname{Li}_{2} S_{x}$ ) and second (peak B: $\mathrm{Li}_{2} \mathrm{~S}_{\mathrm{x}} \rightarrow \mathrm{Li}_{2} \mathrm{~S}_{2} / \mathrm{Li}_{2} \mathrm{~S}$ ) cathodic reduction processes and the anodic oxidation process (peak $\mathrm{C}: \mathrm{Li}_{2} \mathrm{~S}_{2} / \mathrm{Li}_{2} \mathrm{~S} \rightarrow \mathrm{S}_{8}$ ). (d) EIS curves tested at open-circuit voltage for the four different separators, 
inset: equivalent circuit model. (e) Electrical conductivities for the MVS@PP, NVS@PP and HVS@PP separators. (f) Long-term cycling performance of the four different separators at 0.2 C for 300 cycles. (g) Rate performance of the four different separators from $0.1 \mathrm{C}$ to $2 \mathrm{C}$.

To evaluate the electrochemical performance of various separators towards the sulfur cathode, a series of characterizations were further carried out in a coin-type configuration (Figure 3). Firstly, the lithium-ion transference numbers $\left(t_{\mathrm{Li}}{ }^{+}\right)$, defined as the ratio of steady-state current to initial current, of various separators were calculated at a potential of $10 \mathrm{mV} .{ }^{46}$ The $t_{\mathrm{Li}}{ }^{+}$represents the ratio of the total charge carried by lithium ions to that carried by both the lithium ions and the anions in the electrolyte, thus reflecting the lithium-ion transport property of various separators. ${ }^{48,49}$ The $t_{\mathrm{Li}}{ }^{+}$value of the MVS@PP (0.71) and HVS@PP separators (0.69) are comparable to that of the pure PP separator (0.73). NVS@PP, on the other hand, has the lowest lithium-ion transference numbers (0.53). The low $t_{\mathrm{Li}}{ }^{+}$value suggesting the dense NVS functional layer covering on the separator surfaces produced a stronger binding for lithium ions, which is unfavorable to lithium-ion transport and conductivity. ${ }^{46,} 49$ This is also consistent with the results and previous analysis in Figure 2.

Next, cyclic voltammetry (CV) and electrochemical impedance spectra (EIS) were collected to investigate the lithium-ion transfer rates across various separators. Since fast lithium-ion diffusion facilitates the sulfur conversion kinetics in Li-S battery system, the effective lithium-ion diffusion rates of different $\mathrm{VS}_{2}$ separators can be acquired by investigating the $\mathrm{CV}$ curves at various scan rates. For the $\mathrm{CV}$ plots of all the separators (Figure $3 \mathrm{~b}$ and Figure S11), there are two reduction peaks and one oxidation peaks. The first peak (peak A: $\sim 2.3 \mathrm{~V}$ ) and the second peak (peak B: $\sim 2.0 \mathrm{~V}$ ) in the cathodic scan 
represent the reduction of sulfur to soluble $\mathrm{Li}_{2} \mathrm{~S}_{\mathrm{x}}\left(\mathrm{S}_{8} \rightarrow \mathrm{Li}_{2} \mathrm{~S}_{\mathrm{x}}\right)$ and the formation of solid lithium sulfides $\left(\mathrm{Li}_{2} \mathrm{~S}_{\mathrm{x}} \rightarrow \mathrm{Li}_{2} \mathrm{~S}_{2} / \mathrm{Li}_{2} \mathrm{~S}\right)$. The anodic oxidation peak (peak $\mathrm{C}: \sim 2.4 \mathrm{~V}$ ) corresponds to the reversible transition from lithium sulfides to sulfur $\left(\mathrm{Li}_{2} \mathrm{~S}_{2} / \mathrm{Li}_{2} \mathrm{~S} \rightarrow \mathrm{S}_{8}\right)$. According to the Randles-Sevcik equation, ${ }^{30,50}$ the peak current $\left(\mathrm{I}_{\mathrm{p}}\right)$ has a linear relation with the square root of the scan rate $\left(v^{0.5}\right)$ for all separators (Figure S12). The lithium-ion diffusion rate $\left(\mathrm{D}_{\mathrm{Li}}^{+}\right)$can be calculated by the slope of the fitted line $\left(\mathrm{I}_{\mathrm{p}} / v^{0.5}\right)$. As can be concluded from Figure $3 \mathrm{c}$ and Table $\mathrm{S} 1$, the $\mathrm{VS}_{2}$ modified separators at different peaks showed enhanced lithium-ion diffusion rates compared with the pure PP separator. The existed $\mathrm{VS}_{2}$ at the cathode/separator interfaces accelerates the redox process of insulated lithium sulfides and prevents them from depositing in the voids of the separators, ensuring facile lithium-ion diffusion. Benefiting from the high electrolyte affinity and the abundance of active sites, the HVS@PP separator exhibited the fastest lithium-ion diffusion rates among all the $\mathrm{VS}_{2}$ modified separators. Notably, the NVS@PP separator displayed the fastest lithium-ion diffusion at peak A, suggesting the efficient reduction from sulfur to $\mathrm{Li}_{2} \mathrm{~S}_{\mathrm{x}}$. Nevertheless, it could hardly provide enough sites for the reversible conversion of lithium sulfides because of the dense NVS layer, consequently leading to lower lithium-ion diffusion rate at peaks $\mathrm{B}$ and $\mathrm{C}$. The EIS curves of the various separators at open-circuit voltage were further displayed in Figure $3 \mathrm{~d}$, where all the Nyquist plots were composed by a high-frequency semicircle and a low-frequency sloped line, attributing to the charge transfer resistance $\left(R_{c t}\right)$ and mass-diffusion process, respectively. ${ }^{44,} 51$ Based on the fitted equivalent electrical circuit model, the calculated impedance data are summarized in Table S2. The $R_{c t}$ value of the HVS@PP separator $(45.8 \Omega)$ was lower than that of the PP (71.7 $\Omega), \operatorname{MVS} @ P P(56.3 \Omega)$ and NVS@PP (87.2 
$\Omega$ ) separators. This result verified the faster faradic reaction kinetics and smoother charge transfer of the HVS@PP separator, consistent with the above analysis. ${ }^{44,52}$

Beside the lithium-ion transference, the conductivity is another vital factor to affect battery performance. ${ }^{53,54}$ The NVS@PP $\left(2.24 \mathrm{~S} \mathrm{~cm}^{-1}\right)$ and HVS@PP $\left(2.60 \mathrm{~S} \mathrm{~cm}^{-1}\right)$ separators exhibited an almost tenfold higher surface conductivity compared with the MVS@PP separator $\left(0.26 \mathrm{~S} \mathrm{~cm}^{-1}\right)$ (Figure 3e), as the microscale flower-shaped structure of MVS could not uniformly distribute on the PP separator surfaces to ensure continuous electron transfer (Figure S8b). It has been proved that a higher surface conductivity of a separator can lower the cathode/separator interfacial resistance, facilitating low polarization (Figure S13) and fast sulfur conversion during charge/discharge processes. ${ }^{35}$, 54

Following the above-detailed analyses on structure and function, the practical cycling performance of various $\mathrm{VS}_{2}$ modified separators in Li-S batteries was investigated, and the results are shown in Figure 3f. Due to both the fast ion/electron diffusion and effective $\mathrm{Li}_{2} \mathrm{~S}_{\mathrm{x}}$ suppression, the HVS@PP separator delivered the highest initial discharge capacity of $1156 \mathrm{mAh} \mathrm{g}^{-1}$ at $0.2 \mathrm{C}\left(1 \mathrm{C}=1675 \mathrm{mAh} \mathrm{g}^{-1}\right.$ in Li-S battery). The discharge capacity was maintained at $908 \mathrm{mAh} \mathrm{g}^{-1}$ with stabilized Coulombic efficiency $(\sim 99 \%)$ and slow capacity attenuation $(0.072 \%$ per cycle) after 300 cycles. The NVS@PP separator, on the other hand, exhibited the lowest initial discharge capacity of $665 \mathrm{mAh} \mathrm{g}^{-1}$ with a gradual capacity rising in the first 40 cycles derived from the electrochemical activation, ${ }^{20,55,56}$ due to the dense NVS layer that hindered the lithium-ion diffusion/flow. However, the discharge capacity of the NVS@PP separator was retained at $697 \mathrm{mAh} \mathrm{g}^{-1}$ after 300 cycles, higher than that of the MVS@PP (463 mAh g $\left.{ }^{-1}\right)$ and PP (338 mAh g-1) 
separators, proving that suppressing $\mathrm{Li}_{2} \mathrm{~S}_{\mathrm{x}}$ shuttle is more crucial to maintain the stable cycle capacity in Li-S batteries. In addition, the HVS@PP separator also yielded the best rate performance among all separators (Figure 3g). When the current density was increased to $2 \mathrm{C}$, the HVS@PP separator still delivered a high discharge capacity of 630 $\mathrm{mAh} \mathrm{g}^{-1}$, reaffirming the low polarization and the fast reaction kinetics of the HVS@PP separator due to the improved ion/electron transferability at the cathode/separator interface.

A series of post-mortem analyses were further conducted better to evaluate the practical cycling stability of the HVS@PP separator. As shown in Figure S14, the HVS@PP separator still maintained the hexagonal tower-form structure after 300 cycles, proving its high stability in coping with the repeated charging and discharging. EIS plots for the HVS@PP separator after various cycles are also presented in Figure S15, with the corresponding impedance data listed in Table S2. The $R_{c t}$ value of the HVS@PP separator decreased from $45.8 \Omega$ (fresh cell) to $13.5 \Omega$ (after 50 cycles), and $6.3 \Omega$ (after 300 cycles), suggesting a gradually enhanced charge conductivity with the increased number of cycles, which is beneficial to the sulfur redox reaction kinetics in LSBs. ${ }^{7,57}$ The HVS@PP separator also displayed lower $R_{c t}$ values than PP separator after 300 cycles, confirming the superiority of the HVS layer for the fast charge transfer during cycling. Finally, the sulfur content deposited on the lithium metal anodes for the various separators after 300 cycles were quantitatively assessed by inductive coupled plasmaatomic emission spectrometry (ICP-AES) (Figure S16). The lithium metal anode assembled with the HVS@PP separator displayed the lowest sulfur content (5.1 ppm) compared with pure PP, MVS@PP and NVS@PP separators (16.7, 13.4 and 6.7 ppm). 
This excellent $\mathrm{Li}_{2} \mathrm{~S}_{\mathrm{x}}$ suppression ability of the HVS@PP separator once again supports its stable cycling performance shown in Figure 3f.
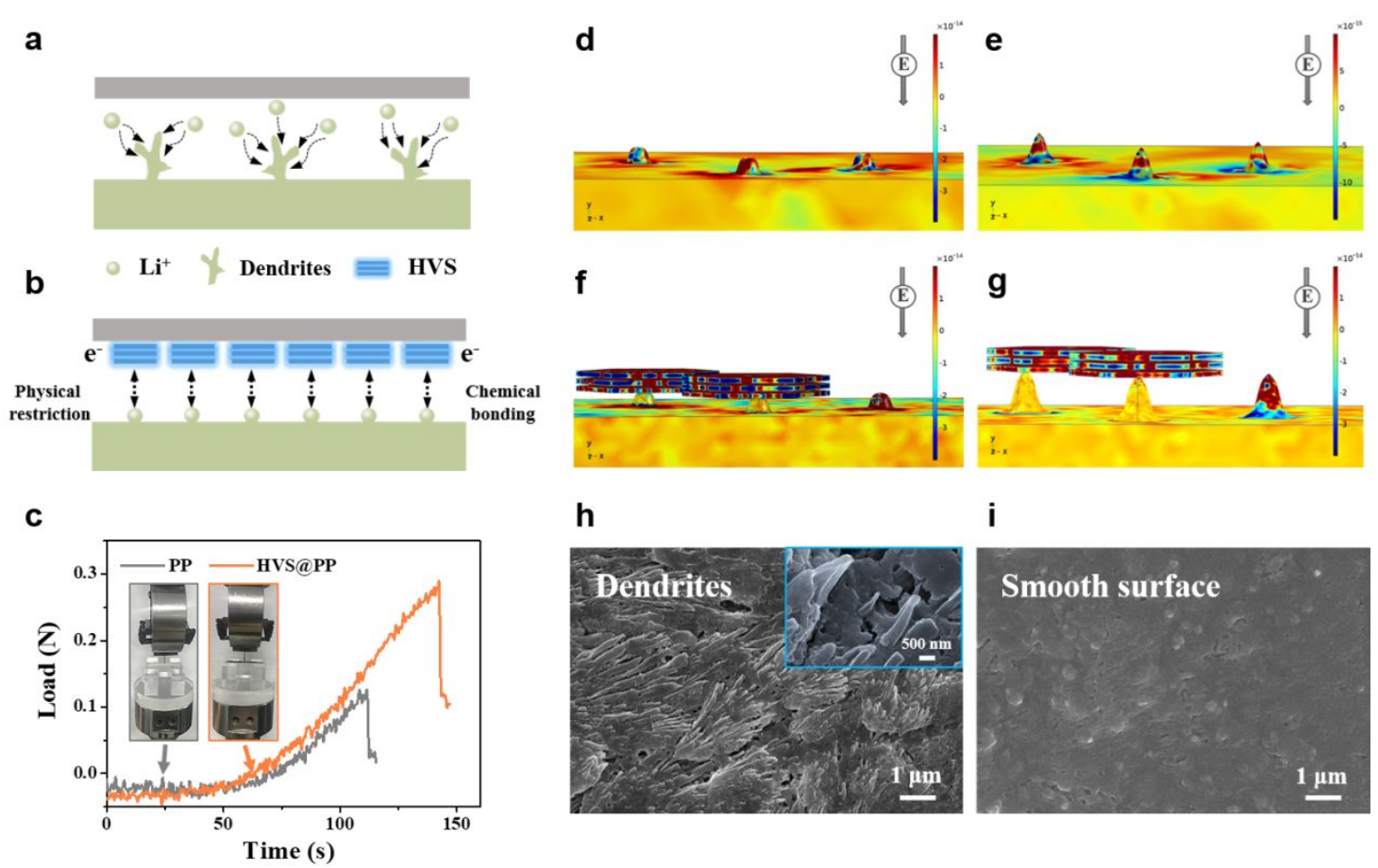

i

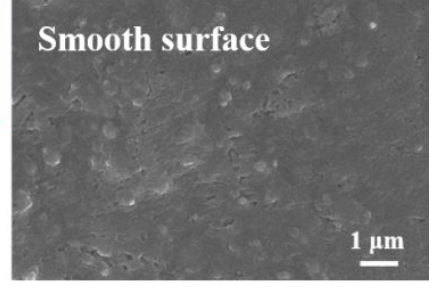

\section{Figure 4. Schematics of lithium growth with different separators on lithium anode}

side. Functional illustrations of the (a) PP and (b) HVS@PP separators on lithium anode side. (c) Puncture strength tests of the PP and HVS@PP separators, inset: the PP and HVS@PP separators were fixed on a sample holder with a gap width of $8 \mathrm{~mm}$ for mechanical puncture tests. Finite element method (FEM) using COMSOL Multiphysics for the simulation of the electric field distribution at different growth periods of ( $\mathrm{d}$ and e) the lithium dendrites and ( $\mathrm{f}$ and $\mathrm{g}$ ) the lithium dendrites covered with the HVS layer. SEM images of the lithium metal anodes with (h) PP and (i) D-HVS@PP separators after stripping/plating for 100 cycles.

Motivated by the excellent performance of the HVS grown on PP (HVS@PP), we then investigated the performance of the double-sided HVS@PP separator for lithium metal anode using various simulation and electrochemical spectroscopic techniques (Figure 4). For clarity, the mechanisms of lithium growth for the three different separators are 
illustrated schematically. For pure PP separator (Figure 4a), it can hardly cope with both the lithium dendrite growth and the volumetric change of bare lithium metal during repeated stripping/plating cycles. Because of the unevenly distributed charges, lithium ions also tend to aggregate near the dendrites and further accelerate the dendrite growth, leading to a severe consumption of electrolyte and the fragmentation of solid electrolyte interphase (SEI) layer. ${ }^{11,} 58$ In contrast, the homogeneous lithium nucleation and growth can be achieved when an HVS layer modifies the surface of the PP separator facing the lithium metal anode for the following reasons (Figure 4b): (a) "Lithiophilic" feature of the HVS (Figure 1c) provides a high chemical affinity with lithium, which can effectively alleviate the dendrite spread and prevent the formation of "dead lithium" during cycling. ${ }^{36,59}$ (b) The stable hexagonal multi-layered nanostructure of the HVS not only accommodate $\mathrm{Li}$ deposition and buffers volume expansion of lithium metal during cycling but also improves the puncture resistance of the PP separator to physically resist the lithium dendrite growth (Figure 4c). ${ }^{18,24,39}$ (c) The improved interfacial conductivity between the separator and the lithium anode ensures homogeneous electric field distribution and decreases the current density, thus conducive to the homogeneous nucleation and the suppression of lithium dendrites growth. ${ }^{38,60}$

To shed more light on the lithiation process, the COMSOL Multiphysics technique was adopted to simulate the electric field distribution at different growth periods of the lithium dendrites with or without an HVS covering layer (Figure 4d-g and Figure S17). ${ }^{59,61}$ For the bare lithium metal anode, the electric field intensity around the dendrites dramatically increases (visible in red) with the extremely uneven electric field distribution. This demonstrates that lithium ions are more likely to be focused at the formed dendrites due 
to the tip effect, further leading to the continuous growth of lithium dendrites. Nevertheless, when the lithium dendrites are covered with the conductive HVS layer, the gathered electric fields around the dendrites are dispersed by the multi-layered HVS structure to form a more uniform distribution on the surface of lithium anode. Hence, the tip effect or hotspot around the lithium dendrites is eliminated.

The lithium metal anodes for different separators were further studied after 100 cycles using SEM (Figure $4 \mathrm{~h}$ and i) and atomic force microscopy (AFM) (Figure S18), to evaluate the practical suppression effect of the D-HVS@PP separator on the lithium dendrites growth. The lithium anode exhibited a quite rough surface with plenty of needle-shaped dendrites when PP separator was used. Conversely, the D-HVS@PP separator maintained a smooth lithium anode surface. The results further confirm the effectiveness of the D-HVS@PP separator in suppressing the dendrite growth as analyzed previously. 
a
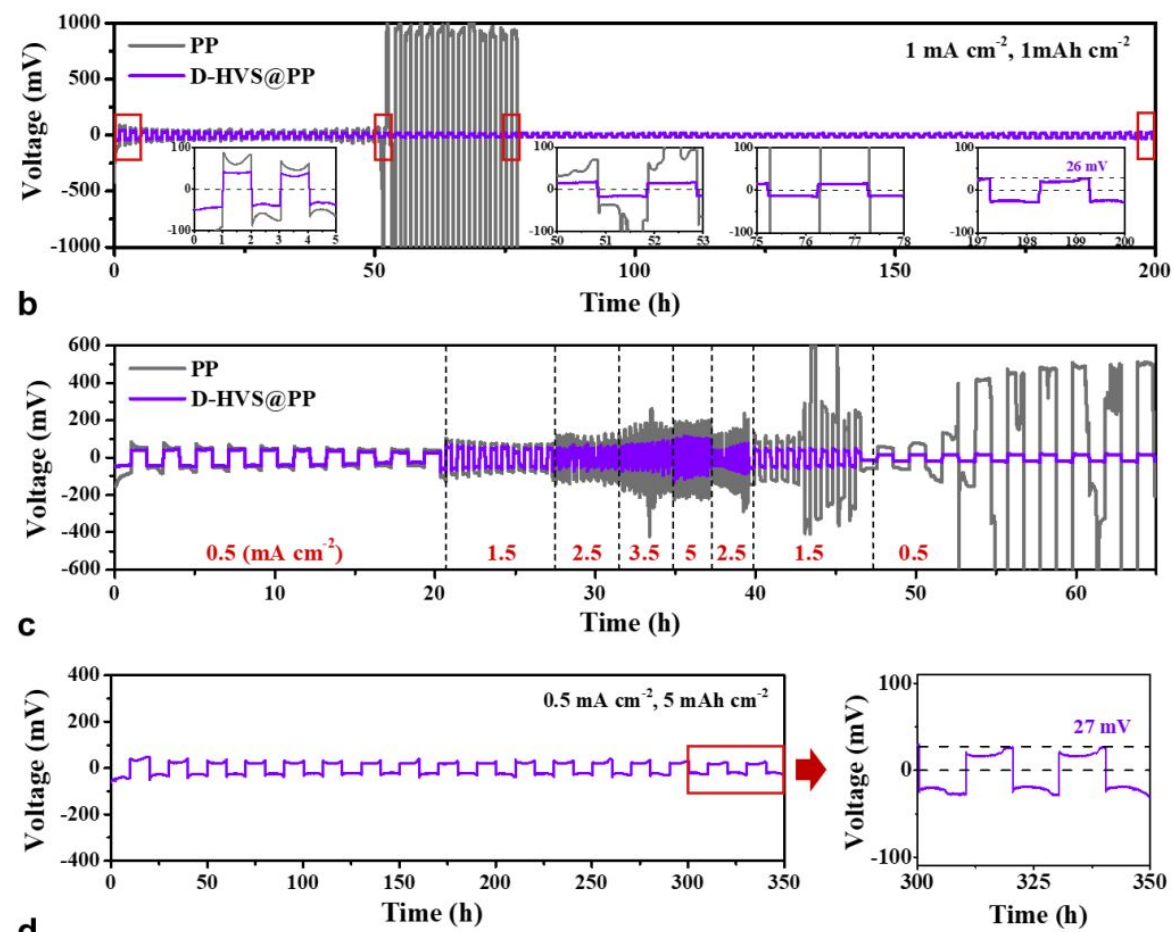

d
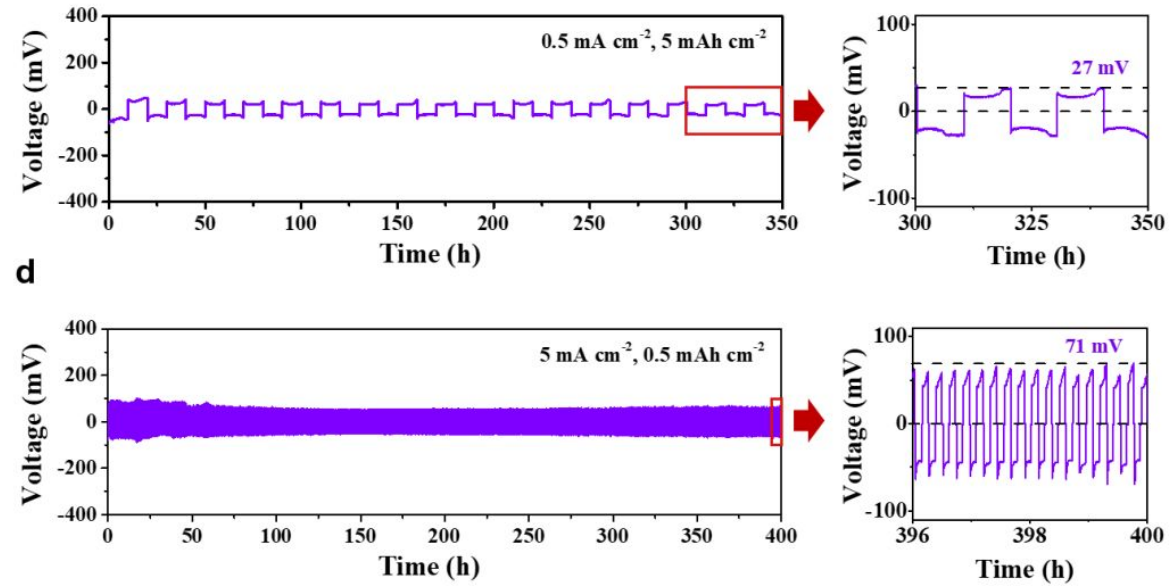

Figure 5. Electrochemical performance towards lithium anode side. (a) The voltage profiles in Li || Li symmetric cells with PP and D-HVS@PP separators at $1 \mathrm{~mA} \mathrm{~cm}{ }^{-2}$ with a stripping/plating capacity of $1 \mathrm{mAh} \mathrm{cm}^{-2}$. (b) Rate performance of the symmetric cells with PP and D-HVS@PP separators at a stripping/plating capacity of $0.5 \mathrm{mAh} \mathrm{cm}^{-2}$. The voltage profiles in Li || Li symmetric cells with D-HVS@PP separator: (c) at $0.5 \mathrm{~mA} \mathrm{~cm}{ }^{-2}$ with a stripping/plating capacity of $5 \mathrm{mAh} \mathrm{cm}-2$ and (d) at $5 \mathrm{~mA} \mathrm{~cm}{ }^{-2}$ with a stripping/plating capacity of $0.5 \mathrm{mAh} \mathrm{cm} \mathrm{cm}^{-2}$.

Galvanostatic cycling performances of $\mathrm{Li} \| \mathrm{Li}$ symmetric cells with the PP and DHVS@PP separators were investigated to evaluate the stability of lithium 
stripping/plating with different separators (Figure 5). Based on the voltage profiles in Figure 5a, the pure PP separator exhibited an initial overpotential of $89 \mathrm{mV}$, twice higher than the D-HVS@PP separator (41 mV). In addition, the polarization for the pure PP separator showed a sharp increase to nearly $1000 \mathrm{mV}$ after $50 \mathrm{~h}$, which might be attributed to the excessive formation of 'dead' lithium on the surface of lithium metal resulting in the fragmentation of SEI layer and unstable Li/electrolyte interface accompanying with poor electrical connection. ${ }^{25,36}$ In contrast, the D-HVS@PP separator maintained a flat lithium stripping/plating plateau with a low overpotential of $26 \mathrm{mV}$ even after $200 \mathrm{~h}$. During the switch of different current densities from $0.5 \mathrm{~mA} \mathrm{~cm}{ }^{-2}$ to $5 \mathrm{~mA}$ $\mathrm{cm}^{-2}$ and reverted to $0.5 \mathrm{~mA} \mathrm{~cm}-2$ (Figure 5b), the D-HVS@PP separator also displayed a more stable polarization vibration compared with the pure PP separator, further indicating its enhanced rate performance in symmetric cells. Even at a larger stripping/plating capacity of $5 \mathrm{mAh} \mathrm{cm}^{-2}$ (Figure $5 \mathrm{c}$ ) or a higher current density of $5 \mathrm{~mA} \mathrm{~cm}^{-2}$ (Figure $5 \mathrm{~d}$ ), the symmetric cells with the D-HVS@PP separator still exhibit stable voltage profiles ( $\sim 27 \mathrm{mV}$ for $350 \mathrm{~h}$ or $\sim 71 \mathrm{mV}$ for $400 \mathrm{~h}$ ). All the results confirm D-HVS@PP separator can facilitate the uniform lithium nucleation and growth due to the synergistic effect of the effective chemical/physical restriction and the improved interfacial conductivity, subsequently achieving the high cycling stability of lithium metal anode. 

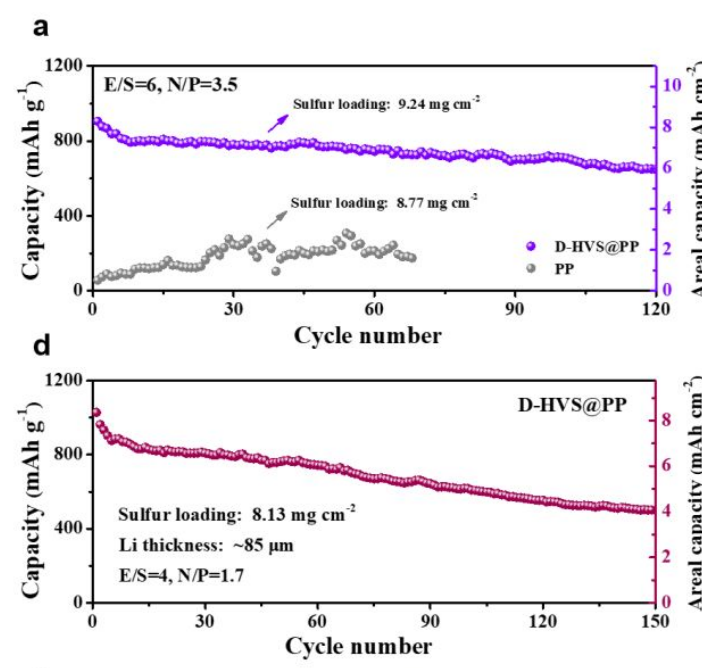

b

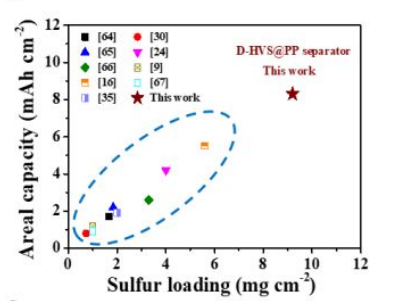

c

e
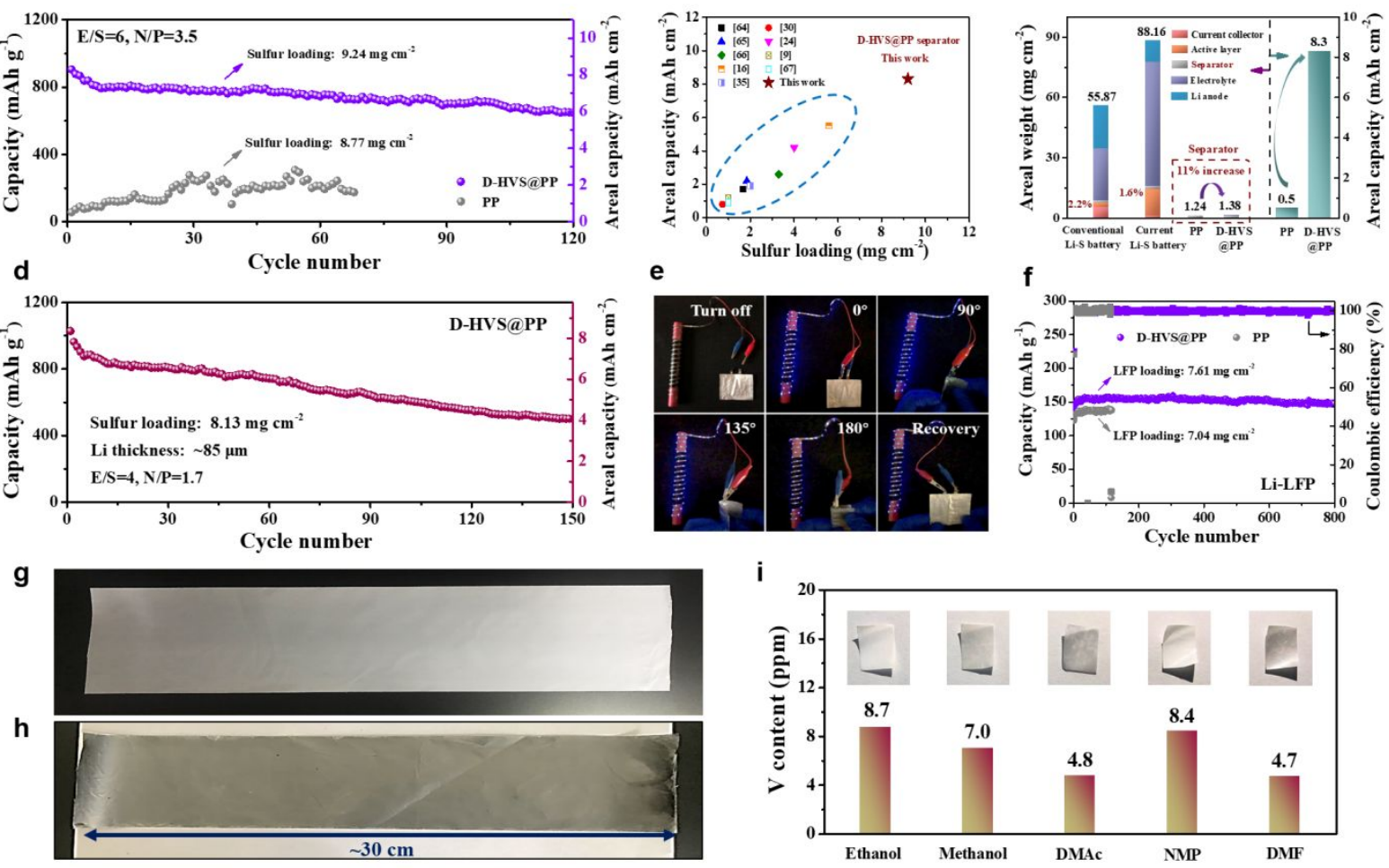

Figure 6. Performance of various battery configurations using different separators. (a)

Cycling performance of PP and D-HVS@PP separators under high sulfur-loading and leanelectrolyte conditions at 0.2 C. (b) Comparison of the areal capacities of D-HVS@PP separator at a high sulfur loading with that of other reported similar materials in Li-S battery, more details are shown in Table S4. (c) Comparison of both the areal weight and areal capacity of PP and DHVS@PP separators. (d) Cycling performance of D-HVS@PP separators under a lower E/S and $\mathrm{N} / \mathrm{P}$ ratios at $0.2 \mathrm{C}$ (e) LEDs illuminating test by a Li-S pouch cell using the D-HVS@PP separator in various folded states. (f) The cycling performance of PP and D-HVS@PP separators in the $\mathrm{Li}_{-}-\mathrm{LiFePO}_{4}$ (LFP) batteries. (g) Image of the commercial PP separator. (h) The large-scale fabrication of D-HVS@PP separator. (i) The vanadium content of the various organic solvents $(10 \mathrm{~mL})$ after the recycling experiments of D-HVS@PP separator probed by ICP-AES, inset: the corresponding separator photos after the recycling treatment. 
Despite numerous efforts made to the development of advanced functional separators, most of the studies focused solely on the high capacity performance and ignored the consideration of sulfur loadings and electrolyte utilization ${ }^{4,20}$. A low sulfur areal loading $\left(<2.0 \mathrm{mg} \mathrm{cm}^{-2}\right)$ and high electrolyte/sulfur $(\mathrm{E} / \mathrm{S})$ ratio $\left(>15 \mathrm{~mL} \mathrm{~g}^{-1}\right)$ can hardly achieve competitive areal capacities and energy density with that of the state-of-art Li-ion batteries ${ }^{20}$. Accordingly, the D-HVS@PP separator with a high sulfur-loading (9.24 mg $\mathrm{cm}^{-2}$ ) S/carbon nanofibers (CNFs) cathode and a $\sim 200 \mu \mathrm{m}$ thick lithium anode (Figure S19a) was employed under a lean-electrolyte condition $\left(6 \mathrm{~mL} \mathrm{~g}^{-1}\right)$ to explore its potential for practical use. The negative to positive capacity ratio (N/P) for this assembled Li-S battery was calculated to be 3.5 (Table S3) ${ }^{62,}{ }^{63}$ Despite the relatively high polarization, which is due to the improved sulfur loading and the low E/S ratio, the D-HVS@PP separator maintained stable charging/discharging platform and excellent areal capacity of $6.0 \mathrm{mAh} \mathrm{cm}^{-2}$ even after 120 cycles. The improved cycle stability can be attributed to the unique amphiphilic property, the high surface conductivity, and the superior electrolyte penetration for $\mathrm{Li}^{+}$transfer (Figure 6a and Figure S20). Notably, the D-HVS@PP has achieved an initial capacity of $8.3 \mathrm{mAh} \mathrm{cm}^{-2}$, which is over 16 times higher than the PP separator $\left(0.5 \mathrm{mAh} \mathrm{cm}^{-2}\right)$ under similar conditions. The high initial area capacity and the excellent cyclic stability of the D-HVS@PP separator also outperforms other similarly reported materials applied as separators, cathodes or interlayers in Li-S batteries (Figure $6 \mathrm{~b}$ and Table S3). $.9,16,24,30,35,64-67$ This performance was achieved just at the expense of an $11 \%$ increase in the separator weight $\left(0.14 \mathrm{mg} \mathrm{cm}^{-2}\right)$, accounting for only $0.24 \%$ in the basic units of conventional Li-S battery or even lower (0.18\%) in the current high sulfurloading battery system (Figure 6c and Table S5). Furthermore, considering that a thinner 
$\mathrm{Li}$ anode and a controlled N/P ratio are more relevant to the practical applications of Li-S batteries, the cycling performance of D-HVS@PP separator was further investigated under more strict conditions; i.e. with a $\sim 85 \mu \mathrm{m}$ thick lithium anode (Figure S19b) and the lower N/P (1.7) and E/S (4 mL g $\left.{ }^{-1}\right)$ ratios (Figure 6d). The battery with D-HVS@PP separator can still deliver a high cell-level gravimetric energy density of $327 \mathrm{Wh} \mathrm{kg}_{\text {cell }}{ }^{-1}$ (the detailed calculations are shown under Table S5) and favourable stability for 150 cycles closed to the battery in Figure 6a. All these results prove the feasibility of the DHVS@PP separator in practical Li-S cells.

Furthermore, to achieve wider application visibility, we verified the separator stability in flexible devices. A Li-S pouch cell was assembled using S/CNFs cathode, D-HVS@PP separator, $\sim 200 \mu \mathrm{m}$ thick lithium anode and vacuum-sealed aluminium-plastic film, with an E/S ratio of $6 \mathrm{~mL} \mathrm{~g}^{-1}$ and an N/P ratio of 3.4. The flexible battery was directly applied in practical light-emitting diodes (LEDs) illumination tests without any additional pressure effect (Figure 6e). This pouch cell exhibited a high open-circuit voltage of 2.45 $\mathrm{V}$ with low self-discharge (Figure S21), and can steadily power an array of LEDs in various folded states from $0^{\circ}$ (flattened) to $180^{\circ}$ (folded) and back to $0^{\circ}$ (flattened), and even when repeatedly bent (Movie S1). Apart from the Li-S battery system, the DHVS@PP separator can also be extended to apply in the $\mathrm{Li}-\mathrm{LiFePO}_{4}$ (LFP) batteries assembled with an LFP cathode and a lithium metal anode (Figure 6f). Due to the high charge conductivity, high electrolyte affinity and homogeneous lithium nucleation, the LiLFP battery with D-HVS@PP separator can deliver a stable discharge capacity of 148.1 $\mathrm{mAh} \mathrm{g}{ }^{-1}$ and areal capacity of $1.13 \mathrm{mAh} \mathrm{cm}^{-2}$ at $1 \mathrm{C}\left(1 \mathrm{C}=170 \mathrm{mAh} \mathrm{g}^{-1}\right.$ for LFP cathode $)$ even after 800 cycles. These values are higher than that recorded for the conventional PP 


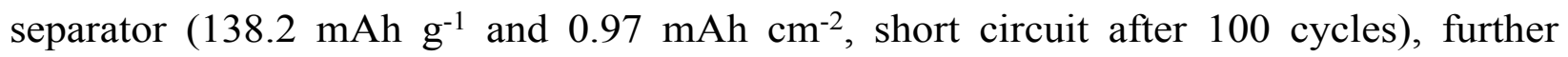
highlighting the potential of the D-HVS@PP separator in broader application fields. Notably, compared with the performance for Li-S batteries in Figure 6 a and $\mathrm{d}$, the lower but more stable cycling performance of Li-LFP battery is attributed to the distinct insertion/extraction lithium storage mechanism of $\mathrm{LiFePO}_{4}$ cathode and its well-known high stability benefiting from the strong support of phosphate group in its lattice structure.

In addition to the universality, the large-scale fabrication and the recyclability of functional materials are other significant factors for practical and commercially viable separator materials. The D-HVS@PP separator can be simply prepared by one-step hydrothermal technique followed by vacuum filtration, hence easy to be commercialized and applied in the mass production of the D-HVS@PP separator. To further proof, we have prepared a thirty-centimetre-long D-HVS@PP separator through a large-area continuous filtration method (Figure $6 \mathrm{~g}$ and $\mathrm{h}$ ). Moreover, considering the cost and toxicity of $\mathrm{VS}_{2}$ materials, ${ }^{68}$ recycling tests were also carried out with the D-HVS@PP separator in various common organic solvents (Figure 6i). The HVS functional materials could be utterly separated from the PP separator to ethanol solvent with an assisted physical vibration, which may be attributed to the higher wettability of ethanol solvent towards PP separator. The recycled vanadium solution can be applied to the reproduction of the D-HVS@PP separator or some other aspects.

\section{CONCLUSIONS}

We designed a double-faces separator based on coating commercial PP membrane with 2D HVS (D-HVS@PP) via a facile and easy to scale up strategy to simultaneously solve different kinds of problems for the practicality of Li-S batteries. The prepared separator was subjected to a 
comprehensive characterization program combining with the DFT calculation and COMSOL Multiphysics simulation. The materials spectroscopic and computational characterization confirmed the excellent physical, chemical, and electrochemical properties of the D-HVS@PP when used as a separator in Li-S batteries, e.g. the "amphiphilic" nature for both $\mathrm{Li}_{2} \mathrm{~S}_{\mathrm{x}}$ and pure lithium, high electronic conductivity and special hexagonal tower-like nanostructure. For the sulfur cathode, the HVS functional layer effectively suppressed the $\mathrm{Li}_{2} \mathrm{~S}_{\mathrm{x}}$ shuttle and ensured the fast interfacial electron transfer and the smooth lithium-ion diffusion through the separator. For the lithium anode, it also promoted the uniform nucleation and growth of lithium and buffered the volume expansion of lithium metal during repeated stripping/plating process. Hence, compared with conventional PP separators, the D-HVS@PP separators enabled a high cell-level gravimetric energy density of $327 \mathrm{Wh} \mathrm{kg}_{\text {cell }}^{-1}$ with stable cycling even under the practically relevant conditions of high sulfur loading, lean-electrolyte and low N/P ratio, or when applied in flexible Li-S pouch and Li-LFP batteries. In particular, the large-scale fabrication and recyclability of the D-HVS@PP separators are also evaluated to highlight its practicality further. We expect this feasible and straightforward separator design can arouse more attention and thoughts to boost the future commercialized development of the Li-S batteries and even other advanced energy storage technologies.

\section{EXPERIMENTAL SECTION}

Chemicals and materials. All chemicals were of analytical grade and used without further purification. Sodium metavanadate $\left(\mathrm{NaVO}_{3}\right)$, sublimed sulfur $(\mathrm{S})$ and N-methyl-2-pyrrolidone (NMP) were obtained from Aladdin. Thioacetamide (TAA) and ammonium hydroxide $\left(\mathrm{NH}_{3} \cdot \mathrm{H}_{2} \mathrm{O}\right)$ were purchased from Sinopharm Chemical Reagent Co. Ltd. Polyvinylidene fluoride 
(PVDF) was obtained from Arkema. Lithium sulfide $\left(\mathrm{Li}_{2} \mathrm{~S}\right)$ was purchased from Sigma-Aldrich. Poly(vinylpyrrolidone) (PVP K90) was supplied by BASF chemical company in Germany.

Preparation of the $\mathbf{V S}_{\mathbf{2}}$ hexagonal nanotowers (HVS). HVS was prepared using a one-step hydrothermal technique. Firstly, $0.468 \mathrm{~g} \mathrm{NaVO}_{3}, 1.503 \mathrm{~g} \mathrm{TAA}, 6 \mathrm{ml} \mathrm{NH} \cdot \mathrm{H}_{2} \mathrm{O}$ and $30 \mathrm{ml}$ distilled water were mixed and magnetically stirred for $5 \mathrm{~min}$. Secondly, $1 \mathrm{~g}$ PVP K90 was added into the mixture; then the solution was stirred for another $40 \mathrm{~min}$ at ambient temperature. The formed precursor solution was then transferred into a $50 \mathrm{ml}$ Teflon-lined stainless-steel autoclave and maintained at $180{ }^{\circ} \mathrm{C}$ for $10 \mathrm{~h}$. After cooling, the precipitate was collected and washed thoroughly with water and ethanol several times to recover the final HVS samples.

Preparation of the $\mathrm{VS}_{2}$ microflowers (MVS) and $\mathrm{VS}_{2}$ nano-bulks (NVS). MVS and NVS samples were prepared for comparison. Conventional MVS were synthesized using the same synthesis method as the HVS without the addition of PVP K90. NVS were obtained by sonicating the as-prepared HVS in ethanol solution for 1 hour.

Preparation of the HVS@PP, MVS@PP and NVS@PP separators. The $\mathrm{VS}_{2}$ modified separators were prepared by direct vacuum filtration technology without using any binder. $2 \mathrm{mg}$ of the as-prepared sample (HVS, MVS or NVS) was dispersed in $20 \mathrm{ml}$ ethanol. The resulting suspensions were directly vacuum filtered onto a commercial PP separator and then dried at 60 ${ }^{\circ} \mathrm{C}$ in a vacuum oven for 6 hours, to form the targeted HVS@PP, MVS@PP and NVS@PP separators, respectively.

Preparation of the sulfur cathodes. The sulfur slurry was prepared by ball-milling a mixture of $280 \mathrm{mg}$ sulfur powder, $80 \mathrm{mg}$ Super P, $40 \mathrm{mg}$ PVDF and $1.8 \mathrm{ml} \mathrm{N}$-methyl-pyrrolidone (NMP) for over 6 hours. The slurry was then coated to an aluminum foil and dried under vacuum at 60 ${ }^{\circ} \mathrm{C}$ for 12 hours to form the sulfur cathode. The sulfur mass loading is $\sim 1.5 \mathrm{mg} \mathrm{cm}^{-2}$. 
Preparation of the $\mathbf{L i}_{2} \mathbf{S}_{6}$ solution. $0.005 \mathrm{M} \mathrm{Li}_{2} \mathrm{~S}_{6}$ solution (30 $\mathrm{mM}$ in sulfur) was obtained by chemically reacting sulfur powder with $\mathrm{Li}_{2} \mathrm{~S}$ in 1,3-dioxolane/1,2-dimethoxyethane solution (DOL/DME, 1:1 by volume).

Characterization. Morphological data and energy dispersive spectra (EDS) mapping were obtained using a field emission scanning electron microscopy (FE-SEM) (GeminiSEM500, China) and a transmission electron microscopy (TEM) (JEOL JEM2100, Japan). X-ray diffraction (XRD) measurements were carried out using a PANalytical X'pert MPDPro (Netherlands) diffractometer with a $\mathrm{Cu}$ Ka radiation source $(40 \mathrm{kV}, 40 \mathrm{~mA})$. Brunauer-EmmettTeller (BET) surface areas and pore size distributions were obtained at $-196{ }^{\circ} \mathrm{C}$ liquid nitrogen temperature) using an ASAP 2020 (America) instrument. X-ray photoelectron spectroscopy (XPS) measurements were carried out on a Kratos Axis Ultra (England) instrument using a monochromatic Al Ka radiation source $(150 \mathrm{~W}, 15 \mathrm{kV}$ and $1486.6 \mathrm{eV})$ at $10^{-9}$ Torr pressure. The contact angle images were obtained on a KRUSS DSA100 (Germany) instrument using Li-S electrolyte as a test liquid. The accurate element contents (sulfur and vanadium) were acquired with an inductively coupled plasma-atomic emission spectrometry (ICP-AES) (Shimadzu ICPE9000, Japan). The electric conductivities of various $\mathrm{VS}_{2}$ modified separators were measured on a four-point probe tester (2182A, America) with a testing current from 4 to $8 \mathrm{~mA}$. Mechanical puncture tests of the separators were carried out on an Instron 5548 (America) Micro Tester Load Test Machine, where a lab-made sample holder with a gap width of $8 \mathrm{~mm}$ was used to fix the tested separators, and the rate of compression displacement was set to be $1 \mathrm{~mm} \mathrm{~min}^{-1}$. A SOLVER NEXT (China) atomic Force Microscopy (AFM) was used to study the dendrite growth on the lithium anode surfaces. 
Cell assembly and electrochemical measurements. Coin-type (2032) and pouch cells were assembled in an Ar-filled glovebox (DELLIX LS750S, China) with moisture and oxygen contents below $1.0 \mathrm{ppm}$. For the Li-S batteries, the sulfur cathode and lithium-metal foil anode were separated by various $\mathrm{VS}_{2}$ modified PP separators. The electrolyte was composed of $1.0 \mathrm{M}$ lithium bis (trifluoromethanesulfonyl) imide (LITFSI) in a solvent mixture of DME/DOL (1 : 1 by volume) with $1.0 \% \mathrm{LiNO}_{3}$ additive. The quantity of electrolyte was controlled at $10 \sim 15 \mu \mathrm{L}$ per $1 \mathrm{mg}$ sulfur. Galvanostatic charge/discharge tests were carried out using a LANHE battery tester within a voltage window of $1.7 \sim 2.8 \mathrm{~V}$ (vs. $\mathrm{Li} / \mathrm{Li}^{+}$). Cyclic voltammetry (CV) was performed using a CHI 660D (China) electrochemical workstation in a voltage range of 1.7 2.8 V. Electrochemical impedance spectra (EIS) were obtained in a frequency range from $0.01 \mathrm{~Hz}$ to $100 \mathrm{kHz}$. In addition, the $\mathrm{Li} \| \mathrm{Li}$ symmetric cells were assembled with the various separators sandwiched between two lithium electrodes. The Li-LFP coin-type batteries were constituted by sandwiching separators between an LFP cathode and a 200 $\mu \mathrm{m}$ thick lithium anode. The electrolyte was 1.0 M LiPF 6 in a solution of the ethylene carbonate and diethyl carbonate (1:1 by weight).

Computational method. The density functional theory (DFT) calculation was performed using the Vienna $A b$-initio Simulation Package. ${ }^{69,70}$ The electron-ion interaction was described by projector augmented-wave (PAW) pseudopotentials. For the exchange and correlation functionals, we use the Perdew-Burke-Ernzerhof (PBE) version of the generalized gradient approximation (GGA) exchange-correlation. ${ }^{70}$ In the DFT calculation, the (011) phase of $\mathrm{VS}_{2}$ and the pure graphene were used to reveal the binding energy with polysulfide (e.g. $\mathrm{Li}_{2} \mathrm{~S}_{4}, \mathrm{Li}_{2} \mathrm{~S}_{6}$ and $\left.\mathrm{Li}_{2} \mathrm{~S}_{8}\right)$. The vacuum layer thickness was set to $15 \AA$ to avoid virtual interaction and obtain more accurate results. The energy cutoff of $400 \mathrm{eV}$ was used for the wave functions expansion. 
The Brillouin zone integration was sampled with a $3 \times 3 \times 1$ k-grid mesh for geometry optimization, and $5 \times 5 \times 1 \mathrm{k}$-grid mesh for electronic properties calculations to achieve high accuracy. The energy and force converged to $1.0 \times 10^{-5} \mathrm{eV}$ atom ${ }^{-1}$ and $0.03 \mathrm{eV} \cdot \AA^{-1}$. The corresponding binding energy $\left(\Delta \mathrm{E}_{\mathrm{BE}}\right)$ is defined as:

$$
\Delta \mathrm{E}_{\mathrm{BE}}=\mathrm{E}_{\mathrm{A}+\mathrm{B}}-\mathrm{E}_{\mathrm{A}}-\mathrm{E}_{\mathrm{B}}
$$

Where the $\mathrm{E}_{\mathrm{A}+\mathrm{B}}$ is the total energy of the structure of the $\mathrm{VS}_{2}(011)$ or graphene combined with polysulfide, $\mathrm{E}_{\mathrm{A}}$ is the total energy of the (011) phase of $\mathrm{VS}_{2}$ or pure graphene, and $\mathrm{E}_{\mathrm{B}}$ is the total energy of the polysulfide (e.g. $\mathrm{Li}_{2} \mathrm{~S}_{4}, \mathrm{Li}_{2} \mathrm{~S}_{6}$ or $\mathrm{Li}_{2} \mathrm{~S}_{8}$ ). Based on the definition, a more negative value indicates a stronger binding system.

Finite element method (FEM) simulation. The electric field distribution in different growth periods of the lithium dendrites covered with or without the HVS layer was performed on a three-dimensional (3D) view by COMSOL Multiphysics 5.3a software. The constructed models referring to size ratio, shape and distribution of materials were established by SolidWorks 2016 according to the experimental characterization results, to make the simulation process as close as possible to the actual situation. To simplify the module process, the physics module of "Electric Current Field" under steady-state conditions was used in the subsequent simulation. The simulated electric field intensity was set to be near platform voltage of $2 \mathrm{~V}$ with the direction from the separator to the anode. The relative dielectric constant of $\mathrm{VS}_{2}$ was set to 3.1, and the conductivity of $\mathrm{VS}_{2}$ was $500 \mathrm{~S} \mathrm{~m}^{-1} \cdot{ }^{71}$ The physical field-controlled grids were selected to be the sequence type and extremely refined cell size.

\section{ASSOCIATED CONTENT}

\section{Supporting Information}


The Supporting Information is available free of charge on the ACS Publications website at DOI: $\quad$ Figures S1 and S2 show the synthetic route and growth mechanism for the HVS. Figures S3-21 show the material characterizations (SEM, TEM, XRD, XPS, AFM and ICPAES), optical images, electrochemical characterizations, calculations and simulations for various samples. Tables S1-4 show some calculated electrochemical performance parameters for various separators in Li-S batteries, including the lithium-ion diffusion rate, EIS resistance, N/P ratio, areal capacity and areal weight.

\title{
Financial Interest Statements
}

The authors declare no conflict of financial interest.

\author{
AUTHOR INFORMATION \\ Corresponding Author \\ *E-mail: dingsj@xjtu.edu.cn (S. Ding). \\ *E-mail: yanwei@xjtu.edu.cn (W. Yan). \\ *E-mail: kx210@cam.ac.uk (K. Xi)
}

\section{Author Contributions}

J. Wang, K. Xi, W. Yan and S. Ding conceived and designed the work; J. Wang, S. Yi, J. Liu, S. Sun, Y. Liu, D. Yang and G. Gao performed the experiments, characterizations, calculations and simulations; J. Wang and K. Xi wrote the manuscript; J. Wang, K. Xi, A. Abdelkader, W. Yan, S. Ding and R. V. Kumar analyzed the data and revised the manuscript. All authors have given approval to the final version of the manuscript.

\section{ACKNOWLEDGMENTS}

This work was supported by the Natural Science Foundation of China (No. 51803164;

51773165), China Postdoctoral Science Foundation (2018M643635), Natural Science 
Foundation of Shaanxi Province (2019JQ-126) and Young Talent Support Plan of Xi'an Jiaotong University. The authors thank Zijun Ren and Chao Li at Instrument Analysis Center of Xi' an Jiaotong University for their assistance with SEM and TEM analysis.

\section{REFERENCES}

1. Griffith, K. J.; Wiaderek, K. M.; Cibin, G.; Marbella, L. E.; Grey, C. P. Niobium Tungsten Oxides for High-Rate Lithium-Ion Energy Storage. Nature 2018, 559, 556-563.

2. $\quad$ Cha, E.; Patel, M. D.; Park, J.; Hwang, J.; Prasad, V.; Cho, K.; Choi, W. 2D MoS 2 as an Efficient Protective Layer for Lithium Metal Anodes in High-Performance Li-S Batteries. Nat. Nanotechnol. 2018, 13, 337-344.

3. Zhang, M.; Xiang, L.; Galluzzi, M.; Jiang, C.; Zhang, S.; Li, J.; Tang, Y. Uniform Distribution of Alloying/Dealloying Stress for High Structural Stability of an Al Anode in HighAreal-Density Lithium-Ion Batteries. Adv. Mater. 2019, 31, 1900826.

4. $\quad$ Pang, Q.; Shyamsunder, A.; Narayanan, B.; Kwok, C. Y.; Curtiss, L. A.; Nazar, L. F. Tuning the Electrolyte Network Structure to Invoke Quasi-Solid State Sulfur Conversion and Suppress Lithium Dendrite Formation in Li-S Batteries. Nature Energy 2018, 3, 783-791.

5. $\quad$ Chang, J.; Shang, J.; Sun, Y.; Ono, L. K.; Wang, D.; Ma, Z.; Huang, Q.; Chen, D.; Liu, G.; Cui, Y.; Qi, Y.; Zheng, Z. Flexible and Stable High-Energy Lithium-Sulfur Full Batteries with Only 100\% Oversized Lithium. Nat. Commun. 2018, 9, 4480.

6. Tsao, Y.; Lee, M.; Miller, E. C.; Gao, G.; Park, J.; Chen, S.; Katsumata, T.; Tran, H.; Wang, L.-W.; Toney, M. F.; Cui, Y.; Bao, Z. Designing a Quinone-Based Redox Mediator to Facilitate $\mathrm{Li}_{2} \mathrm{~S}$ Oxidation in Li-S Batteries. Joule 2019, 3, 872-884.

7. Wang, J.; Yang, G.; Chen, J.; Liu, Y.; Wang, Y.; Lao, C. Y.; Xi, K.; Yang, D.; Harris, C. J.; Yan, W.; Ding, S.; Kumar, R. V. Flexible and High-Loading Lithium-Sulfur Batteries Enabled by Integrated Three-In-One Fibrous Membranes. Adv. Energy Mater. 2019, 9, 1902001. 8. Xu, G.; Kushima, A.; Yuan, J.; Dou, H.; Xue, W.; Zhang, X.; Yan, X.; Li, J. Ad Hoc Solid Electrolyte on Acidized Carbon Nanotube Paper Improves Cycle Life of Lithium-Sulfur Batteries. Energy Environ. Sci. 2017, 10, 2544-2551. 
9. Xi, K.; He, D.; Harris, C.; Wang, Y.; Lai, C.; Li, H.; Coxon, P. R.; Ding, S.; Wang, C.; Kumar, R. V. Enhanced Sulfur Transformation by Multifunctional $\mathrm{FeS}_{2} / \mathrm{FeS} / \mathrm{S}$ Composites for High-Volumetric Capacity Cathodes in Lithium-Sulfur Batteries. Adv. Sci. 2019, 6, 1800815.

10. Lin, D.; Liu, Y.; Cui, Y. Reviving the Lithium Metal Anode for High-Energy Batteries. Nat. Nanotechnol. 2017, 12, 194-206.

11. Cheng, X. B.; Zhang, R.; Zhao, C. Z.; Zhang, Q. Toward Safe Lithium Metal Anode in Rechargeable Batteries: A Review. Chem. Rev. 2017, 117, 10403-10473.

12. Lin, H.; Yang, L.; Jiang, X.; Li, G.; Zhang, T.; Yao, Q.; Zheng, G. W.; Lee, J. Y. Electrocatalysis of Polysulfide Conversion by Sulfur-Deficient $\mathrm{MoS}_{2}$ Nanoflakes for LithiumSulfur Batteries. Energy Environ. Sci. 2017, 10, 1476-1486.

13. Xi, K.; Chen, B.; Li, H.; Xie, R.; Gao, C.; Zhang, C.; Kumar, R. V.; Robertson, J. Soluble Polysulphide Sorption Using Carbon Nanotube Forest for Enhancing Cycle Performance in a Lithium-Sulphur Battery. Nano Energy 2015, 12, 538-546.

14. Gu, X.; Wang, Y.; Lai, C.; Qiu, J.; Li, S.; Hou, Y.; Martens, W.; Mahmood, N.; Zhang, S. Microporous Bamboo Biochar for Lithium-Sulfur Batteries. Nano Res. 2014, 8, 129-139.

15. Chen, H.; Ling, M.; Hencz, L.; Ling, H. Y.; Li, G.; Lin, Z.; Liu, G.; Zhang, S. Exploring Chemical, Mechanical, and Electrical Functionalities of Binders for Advanced Energy-Storage Devices. Chem. Rev. 2018, 118, 8936-8982.

16. He, J.; Chen, Y.; Manthiram, A. Vertical $\mathrm{Co}_{9} \mathrm{~S}_{8}$ Hollow Nanowall Arrays Grown on a Celgard Separator as a Multifunctional Polysulfide Barrier for High-Performance Li-S Batteries. Energy Environ. Sci. 2018, 11, 2560-2568.

17. Li, G.; Wang, C.; Cai, W.; Lin, Z.; Li, Z.; Zhang, S. The Dual Actions of Modified Polybenzimidazole in Taming the Polysulfide Shuttle for Long-Life Lithium-Sulfur Batteries. NPG Asia Mater. 2016, 8, e317-e317.

18. Liu, S.; Xia, X.; Zhong, Y.; Deng, S.; Yao, Z.; Zhang, L.; Cheng, X.-B.; Wang, X.; Zhang, Q.; Tu, J. 3D TiC/C Core/Shell Nanowire Skeleton for Dendrite-Free and Long-Life Lithium Metal Anode. Adv. Energy Mater. 2018, 8, 1702322.

19. Dong, J.; Dai, H.; Fan, Q.; Lai, C.; Zhang, S. Grain Refining Mechanisms: Initial Levelling Stage During Nucleation for High-Stability Lithium Anodes. Nano Energy 2019, 66, 104128. 
20. Peng, H.-J.; Huang, J.-Q.; Cheng, X.-B.; Zhang, Q. Review on High-Loading and HighEnergy Lithium-Sulfur Batteries. Adv. Energy Mater. 2017, 7, 1700260.

21. Bai, S.; Liu, X.; Zhu, K.; Wu, S.; Zhou, H. Metal-Organic Framework-Based Separator for Lithium-Sulfur Batteries. Nature Energy 2016, 1, 16094.

22. Lee, Y.-H.; Kim, J.-H.; Kim, J.-H.; Yoo, J.-T.; Lee, S.-Y. Spiderweb-Mimicking AnionExchanging Separators for Li-S Batteries. Adv. Funct. Mater. 2018, 28, 1801422.

23. Chang, Z.; Qiao, Y.; Wang, J.; Deng, H.; He, P.; Zhou, H. Fabricating Better MetalOrganic Frameworks Separators for Li-S Batteries: Pore Sizes Effects Inspired Channel Modification Strategy. Energy Storage Mater. 2020, 25, 164-171.

24. Wu, J.; Zeng, H.; Li, X.; Xiang, X.; Liao, Y.; Xue, Z.; Ye, Y.; Xie, X. Ultralight Layer-By-Layer Self-Assembled $\mathrm{MoS}_{2}$-Polymer Modified Separator for Simultaneously Trapping Polysulfides and Suppressing Lithium Dendrites. Adv. Energy Mater. 2018, 8, 1802430.

25. He, Y.; Chang, Z.; Wu, S.; Qiao, Y.; Bai, S.; Jiang, K.; He, P.; Zhou, H. Simultaneously Inhibiting Lithium Dendrites Growth and Polysulfides Shuttle by a Flexible MOF-Based Membrane in Li-S Batteries. Adv. Energy Mater. 2018, 8, 1802130.

26. Li, Y. J.; Wang, C.; Wang, W. Y.; Eng, A. Y. S.; Wan, M. T.; Fu, L.; Mao, E. Y.; Li, G. C.; Tang, J.; Seh, Z. W.; Sun, Y. M. Enhanced Chemical Immobilization and Catalytic Conversion of Polysulfide Intermediates Using Metallic Mo Nanoclusters for High-Performance Li-S Batteries. ACS Nano 2020, 14, 1148-1157.

27. Li, M.; Wan, Y.; Huang, J.-K.; Assen, A. H.; Hsiung, C.-E.; Jiang, H.; Han, Y.; Eddaoudi, M.; Lai, Z.; Ming, J.; Li, L.-J. Metal-Organic Framework-Based Separators for Enhancing Li-S Battery Stability: Mechanism of Mitigating Polysulfide Diffusion. ACS Energy Lett. 2017, 2, 2362-2367.

28. Kim, J.-H.; Lee, Y.-H.; Cho, S.-J.; Gwon, J.-G.; Cho, H.-J.; Jang, M.; Lee, S.-Y.; Lee, S.Y. Nanomat Li-S Batteries Based on All-Fibrous Cathode/Separator Assemblies and Reinforced Li Metal Anodes: Towards Ultrahigh Energy Density and Flexibility. Energy Environ. Sci. 2019, 12, 177-186.

29. Pan, Y.; Chou, S.; Liu, H. K.; Dou, S. X. Functional Membrane Separators for NextGeneration High-Energy Rechargeable Batteries. Natl. Sci. Rev. 2017, 4, 917-933. 
30. Zhou, G.; Tian, H.; Jin, Y.; Tao, X.; Liu, B.; Zhang, R.; Seh, Z. W.; Zhuo, D.; Liu, Y.;

Sun, J.; Zhao, J.; Zu, C.; Wu, D. S.; Zhang, Q.; Cui, Y. Catalytic Oxidation of $\mathrm{Li}_{2} \mathrm{~S}$ on the Surface of Metal Sulfides for Li-S Batteries. P. Natl. Acad. Sci. 2017, 114, 840-845.

31. Chen, X.; Peng, H.-J.; Zhang, R.; Hou, T.-Z.; Huang, J.-Q.; Li, B.; Zhang, Q. An Analogous Periodic Law for Strong Anchoring of Polysulfides on Polar Hosts in Lithium Sulfur Batteries: S- or Li-Binding on First-Row Transition-Metal Sulfides? ACS Energy Lett. 2017, 2, 795-801.

32. Sun, R.; Wei, Q.; Sheng, J.; Shi, C.; An, Q.; Liu, S.; Mai, L. Novel Layer-By-Layer Stacked $\mathrm{VS}_{2}$ Nanosheets with Intercalation Pseudocapacitance for High-Rate Sodium Ion Charge Storage. Nano Energy 2017, 35, 396-404.

33. Yan, J.; Liu, F.; Hu, Z.; Gao, J.; Zhou, W.; Huo, H.; Zhou, J.; Li, L. Realizing DendriteFree Lithium Deposition with a Composite Separator. Nano Lett. 2020, 20, 3798-3807.

34. Fu, C.; Venturi, V.; Kim, J.; Ahmad, Z.; Ells, A. W.; Viswanathan, V.; Helms, B. A. Universal Chemomechanical Design Rules for Solid-Ion Conductors to Prevent Dendrite Formation in Lithium Metal Batteries. Nat. Mater. 2020, DOI: 10.1038/s41563-020-0655-2.

35. Sun, J.; Sun, Y.; Pasta, M.; Zhou, G.; Li, Y.; Liu, W.; Xiong, F.; Cui, Y. Entrapment of Polysulfides by a Black-Phosphorus-Modified Separator for Lithium-Sulfur Batteries. Adv. Mater. 2016, 28, 9797-9803.

36. Ye, H.; Zheng, Z. J.; Yao, H. R.; Liu, S. C.; Zuo, T. T.; Wu, X. W.; Yin, Y. X.; Li, N. W.; Gu, J. J.; Cao, F. F.; Guo, Y. G. Guiding Uniform Li Plating/Stripping through LithiumAluminum Alloying Medium for Long-Life Li Metal Batteries. Angew. Chem. Int. Ed. 2019, 58, 1094-1099.

37. Oh, J.; Jo, H.; Lee, H.; Kim, H.-T.; Lee, Y. M.; Ryou, M.-H. Polydopamine-Treated Three-Dimensional Carbon Fiber-Coated Separator for Achieving High-Performance Lithium Metal Batteries. J. Power Sources 2019, 430, 130-136.

38. Zhang, R.; Cheng, X. B.; Zhao, C. Z.; Peng, H. J.; Shi, J. L.; Huang, J. Q.; Wang, J.; Wei, F.; Zhang, Q. Conductive Nanostructured Scaffolds Render Low Local Current Density to Inhibit Lithium Dendrite Growth. Adv. Mater. 2016, 28, 2155-2162. 
39. Luo, J.; Wang, C.; Wang, H.; Hu, X.; Matios, E.; Lu, X.; Zhang, W.; Tao, X.; Li, W. Pillared Mxene with Ultralarge Interlayer Spacing as a Stable Matrix for High Performance Sodium Metal Anodes. Adv. Funct. Mater. 2019, 29, 1805946.

40. Koczkur, K. M.; Mourdikoudis, S.; Polavarapu, L.; Skrabalak, S. E. Polyvinylpyrrolidone (PVP) in Nanoparticle Synthesis. Dalton Trans. 2015, 44, 17883-17905.

41. Fang, Z.; Hao, S.; Long, L.; Fang, H.; Qiang, T.; Song, Y. The Enhanced Photoelectrochemical Response of $\mathrm{SnSe}_{2}$ Nanosheets. CrystEngComm 2014, 16, 2404.

42. Chen, J. S.; Liu, J.; Qiao, S. Z.; Xu, R.; Lou, X. W. Formation of Large 2D Nanosheets via PVP-Assisted Assembly of Anatase $\mathrm{TiO}_{2}$ Nanomosaics. Chem. Commun. 2011, 47, 10443 10445 .

43. Lai, C.; Wu, Z.; Gu, X.; Wang, C.; Xi, K.; Kumar, R. V.; Zhang, S. Reinforced Conductive Confinement of Sulfur for Robust and High-Performance Lithium-Sulfur Batteries. ACS Appl. Mater. Interfaces 2015, 7, 23885-23892.

44. Shao, H.; Ai, F.; Wang, W.; Zhang, H.; Wang, A.; Feng, W.; Huang, Y. Crab ShellDerived Nitrogen-Doped Micro-/Mesoporous Carbon as an Effective Separator Coating for High Energy Lithium-Sulfur Batteries. J. Mater. Chem. A 2017, 5, 19892-19900.

45. Ling, Z.; Ren, C. E.; Zhao, M. Q.; Yang, J.; Giammarco, J. M.; Qiu, J.; Barsoum, M. W.; Gogotsi, Y. Flexible and Conductive Mxene Films and Nanocomposites with High Capacitance. P. Natl. Acad. Sci. 2014, 111, 16676-16681.

46. Ghazi, Z. A.; He, X.; Khattak, A. M.; Khan, N. A.; Liang, B.; Iqbal, A.; Wang, J.; Sin, H.; Li, L.; Tang, Z. $\mathrm{MoS}_{2} /$ Celgard Separator as Efficient Polysulfide Barrier for Long-Life Lithium-Sulfur Batteries. Adv. Mater. 2017, 29, 1606817.

47. Zhang, D.; Wang, S.; Li, B.; Gong, Y.; Yang, S. Horizontal Growth of Lithium on Parallelly Aligned Mxene Layers towards Dendrite-Free Metallic Lithium Anodes. Adv. Mater. 2019, 31, 1901820.

48. Chen, L.; Li, W.; Fan, L. Z.; Nan, C. W.; Zhang, Q. Intercalated Electrolyte with High Transference Number for Dendrite-Free Solid-State Lithium Batteries. Adv. Funct. Mater. 2019, 29, 1901047.

49. Wang, Z.; Huang, W.; Hua, J.; Wang, Y.; Yi, H.; Zhao, W.; Zhao, Q.; Jia, H.; Fei, B.; Pan, F. An Anionic-MOF-Based Bifunctional Separator for Regulating Lithium Deposition and 
Suppressing Polysulfides Shuttle in Li-S Batteries. Small Methods 2020, DOI: 10.1002/smtd.202000082, 2000082.

50. Wang, Y.; Zhang, R.; Chen, J.; Wu, H.; Lu, S.; Wang, K.; Li, H.; Harris, C. J.; Xi, K.; Kumar, R. V.; Ding, S. Enhancing Catalytic Activity of Titanium Oxide in Lithium-Sulfur Batteries by Band Engineering. Adv. Energy Mater. 2019, 9, 1900953.

51. Yu, M.; Wang, Z.; Wang, Y.; Dong, Y.; Qiu, J. Freestanding Flexible Li $2 \mathrm{~S}$ Paper Electrode with High Mass and Capacity Loading for High-Energy Li-S Batteries. Adv. Energy Mater. 2017, 7, 1700018.

52. Cherian, C. T.; Sundaramurthy, J.; Reddy, M. V.; Suresh Kumar, P.; Mani, K.; Pliszka, D.; Sow, C. H.; Ramakrishna, S.; Chowdari, B. V. Morphologically Robust $\mathrm{NiFe}_{2} \mathrm{O}_{4}$ Nanofibers as High Capacity Li-Ion Battery Anode Material. ACS Appl. Mater. Interfaces 2013, 5, 9957.

53. Sun, L.; Wang, D.; Luo, Y.; Wang, K.; Kong, W.; Wu, Y.; Zhang, L.; Jiang, K.; Li, Q.; Zhang, Y.; Wang, J.; Fan, S. Sulfur Embedded in a Mesoporous Carbon Nanotube Network as a Binder-Free Electrode for High-Performance Lithium-Sulfur Batteries. ACS Nano 2016, 10, 1300-1308.

54. Wang, H.; Zhang, W.; Liu, H.; Guo, Z. A Strategy for Configuration of an Integrated Flexible Sulfur Cathode for High-Performance Lithium-Sulfur Batteries. Angew. Chem. Int. Ed. 2016, 55, 3992-3996.

55. Wang, J.; Yang, G.; Wang, L.; Yan, W. Synthesis of One-Dimensional $\mathrm{NiFe}_{2} \mathrm{O}_{4}$ Nanostructures: Tunable Morphology and High-Performance Anode Materials for Li Ion Batteries. J. Mater. Chem. A 2016, 4, 8620-8629.

56. Mao, Y.; Li, G.; Guo, Y.; Li, Z.; Liang, C.; Peng, X.; Lin, Z. Foldable Interpenetrated Metal-Organic Frameworks/Carbon Nanotubes Thin Film for Lithium-Sulfur Batteries. Nat. Commun. 2017, 8, 14628.

57. Wang, L.; He, Y.-B.; Shen, L.; Lei, D.; Ma, J.; Ye, H.; Shi, K.; Li, B.; Kang, F. UltraSmall Self-Discharge and Stable Lithium-Sulfur Batteries Achieved by Synergetic Effects of Multicomponent Sandwich-Type Composite Interlayer. Nano Energy 2018, 50, 367-375.

58. Zhang, R.; Li, N. W.; Cheng, X. B.; Yin, Y. X.; Zhang, Q.; Guo, Y. G. Advanced Micro/Nanostructures for Lithium Metal Anodes. Adv. Sci. 2017, 4, 1600445. 
59. Liu, B.; Zhang, Y.; Pan, G.; Ai, C.; Deng, S.; Liu, S.; Liu, Q.; Wang, X.; Xia, X.; Tu, J. Ordered Lithiophilic Sites to Regulate Li Plating/Stripping Behavior for Superior Lithium Metal Anodes. J. Mater. Chem. A 2019, 7, 21794-21801.

60. Cheng, X.-B.; Peng, H.-J.; Huang, J.-Q.; Zhang, R.; Zhao, C.-Z.; Zhang, Q. Dual-Phase Lithium Metal Anode Containing a Polysulfide-Induced Solid Electrolyte Interphase and Nanostructured Graphene Framework for Lithium-Sulfur Batteries. ACS Nano 2015, 9, 63736382.

61. Wang, A.; Deng, Q.; Deng, L.; Guan, X.; Luo, J. Eliminating Tip Dendrite Growth by Lorentz Force for Stable Lithium Metal Anodes. Adv. Funct. Mater. 2019, 29, 1902630.

62. Abe, Y.; Kumagai, S. Effect of Negative/Positive Capacity Ratio on the Rate and Cycling

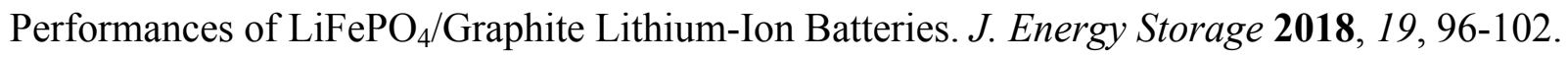

63. Lu, K.; Liu, Y.; Chen, J.; Zhang, Z.; Cheng, Y. Redox Catalytic and Quasi-Solid Sulfur Conversion for High-Capacity Lean Lithium Sulfur Batteries. ACS Nano 2019, 13, 14540-14548. 64. Wu, H.; Huan, Y.; Wang, D.; Li, M.; Cheng, X.; Bai, Z.; Wu, P.; Peng, W.; Zhang, R.; Ji, Z.; Zou, M.; Yan, X. Hierarchical VS $_{2}$ Nano-Flowers as Sulfur Host for Lithium Sulfur Battery Cathodes. J. Electrochem. Soc. 2019, 166, A188-A194.

65. Cheng, Z.; Xiao, Z.; Pan, H.; Wang, S.; Wang, R. Elastic Sandwich-Type RGO-VS $2 / S$ Composites with High Tap Density: Structural and Chemical Cooperativity Enabling LithiumSulfur Batteries with High Energy Density. Adv. Energy Mater. 2018, 8, 1702337.

66. Guo, Y.; Zhang, Y.; Zhang, Y.; Xiang, M.; Wu, H.; Liu, H.; Dou, S. Interwoven $\mathrm{V}_{2} \mathrm{O}_{5}$ Nanowire/Graphene Nanoscroll Hybrid Assembled as Efficient Polysulfide-TrappingConversion Interlayer for Long-Life Lithium-Sulfur Batteries. J. Mater. Chem. A 2018, 6, 19358-19370.

67. Dong, Y.; Zheng, S.; Qin, J.; Zhao, X.; Shi, H.; Wang, X.; Chen, J.; Wu, Z. S. AllMxene-Based Integrated Electrode Constructed by $\mathrm{Ti}_{3} \mathrm{C}_{2}$ Nanoribbon Framework Host and Nanosheet Interlayer for High-Energy-Density Li-S Batteries. ACS Nano 2018, 12, 2381-2388.

68. Feng, J.; Sun, X.; Wu, C.; Peng, L.; Lin, C.; Hu, S.; Yang, J.; Xie, Y. Metallic FewLayered $\mathrm{VS}_{2}$ Ultrathin Nanosheets: High Two-Dimensional Conductivity for In-Plane Supercapacitors. J. Am. Chem. Soc. 2011, 133, 17832-17838.

69. Kresse, G.; Furthmüller, J. Efficient Iterative Schemes for Ab Initio Total-Energy Calculations Using a Plane-Wave Basis Set. Phys. Rev. B 1996, 54, 11169-11186. 
70. Kresse, G.; Hafner, J. Ab Initio Molecular-Dynamics Simulation of the Liquid-MetalAmorphous-Semiconductor Transition in Germanium. Phys. Rev. B 1994, 49, 14251-14269.

71. Yu, D.; Pang, Q.; Gao, Y.; Wei, Y.; Wang, C.; Chen, G.; Du, F. Hierarchical Flower-Like $\mathrm{VS}_{2}$ Nanosheets-a High Rate-Capacity and Stable Anode Material for Sodium-Ion Battery. Energy Storage Mater. 2018, 11, 1-7. 


\section{For Table of Contents Use Only}

Suppressing the Shuttle Effect and Dendrite Growth in Lithium-Sulfur Batteries

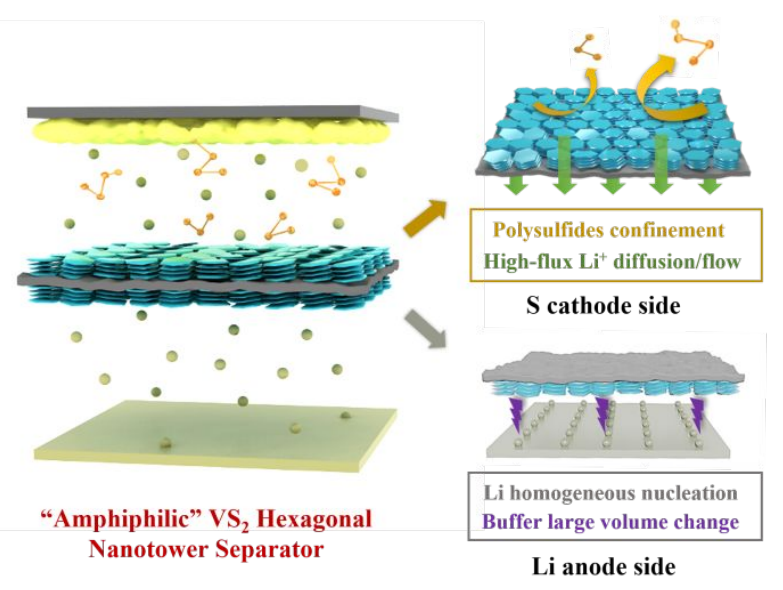

